\title{
Efficient Computation of Semivalues for Game-Theoretic Network Centrality
}

\author{
Mateusz K. Tarkowski \\ Department of Computer Science, University of Oxford \\ OX1 3QD, United Kingdom \\ Piotr L. Szczepański \\ Nykredit / BEC Poland \\ 00-113 Warsaw, Poland \\ Tomasz P. Michalak \\ Institute of Informatics, University of Warsaw \\ 02-097 Warsaw, Poland \\ Paul Harrenstein \\ Department of Computer Science, University of Oxford \\ OX1 3QD, United Kingdom \\ Michael Wooldridge \\ Department of Computer Science, University of Oxford \\ OX1 3QD, United Kingdom
}

MATEUSZTARKOWSKI@GMAIL.COM

Q1N9@NYKREDIT.DK

TPM@MIMUW.EDU.PL

PAUL.HARRENSTEIN@CS.OX.AC.UK

MICHAEL.WOOLDRIDGE@CS.OX.AC.UK

\begin{abstract}
Some game-theoretic solution concepts such as the Shapley value and the Banzhaf index have recently gained popularity as measures of node centrality in networks. While this direction of research is promising, the computational problems that surround it are challenging and have largely been left open. To date there are only a few positive results in the literature, which show that some game-theoretic extensions of degree-, closeness- and betweenness-centrality measures are computable in polynomial time, i.e., without the need to enumerate the exponential number of all possible coalitions. In this article, we show that these results can be extended to a much larger class of centrality measures that are based on a family of solution concepts known as semivalues. The family of semivalues includes, among others, the Shapley value and the Banzhaf index. To this end, we present a generic framework for defining game-theoretic network centralities and prove that all centrality measures that can be expressed in this framework are computable in polynomial time. Using our framework, we present a number of new and polynomial-time computable game-theoretic centrality measures.
\end{abstract}

\section{Introduction}

Determining the significance or centrality of a node or edge in a network is an important research topic, the modern approach to which goes back to at least the work of Moreno and Jennings on the inmates of a prison and the residents of a reform school for girls (Moreno, 1932, 1934; Freeman, 2004). The problem has been extensively studied in biology, social network analysis, and computer science, where it has led to such diverse applications as, for instance, identifying proteins that are critical for the survival of cells (Jeong, Mason, Barabasi, \& Oltvai, 2001), the maximisation of 
influence in social networks (Kempe, Kleinberg, \& Tardos, 2003), and ranking websites in order to improve web search result accuracy (Page, Brin, Motwani, \& Winograd, 1999).

In the literature, a wide range of centrality measures have been proposed, each presenting a different perspective on how to quantify the centrality of a node or edge in a network. Among these, the four most fundamental and prominent are degree centrality, closeness centrality, betweenness centrality, and eigenvector centrality (Bonacich, 1972; Freeman, 1979). Degree centrality ranks nodes according to the number of direct connections they have to other nodes, i.e., their degree: nodes with more direct connections are ranked higher than those that have fewer. By contrast, closeness centrality considers the distances between nodes: if, on average, a node is close to all other nodes in the network, then it will be ranked highly. Finally, betweenness centrality considers the shortest paths between any pair of nodes in the network: the larger the number of these paths a node lies on, the higher its ranking.

A common feature of classic centrality measures is that they focus on individual nodes, without taking into account the various synergies that may occur when nodes are considered in groups. Such synergies are crucial for a variety of applications. For example, if we consider an epidemic spreading through a city, it is desirable to isolate those who are infected from those who are not. This can be achieved both through quarantine and vaccination. Although it is often not feasible to quarantine and vaccinate everyone in a short period of time, it may be possible to vaccinate those who act as bridges between infected and healthy groups of people. In effect, this can disconnect one (infected) part of the network from another (healthy) part. Even when vaccinating a single node cannot achieve this, vaccinating a group may. We can thus say, that vaccinating groups achieves a type of positive synergy. Yet, some groups are more effective for this purpose. Hence, it would seem natural that groups themselves also should be ranked according to their importance.

Recognizing the importance of this observation, Everett and Borgatti (1999) were the first to propose extensions of centrality measures to groups of nodes. In particular, they defined group degree centrality, group betweenness centrality, and group closeness centrality, each of which ranks groups of nodes based on their combined performance. This enables us to address in a principled fashion such questions as: which group is most central, or how important is a certain arm of a larger institution (e.g., a department of a university).

In view of these considerations, it has been argued that the centrality of a single node should not only depend on individual traits like degree, closeness, or betweenness, but also on the centrality of the groups of nodes it belongs to. Pursuing this line of thought, the centrality of individual nodes is a function of the centralities of groups of nodes. In a broader setting, the issue of how to aggregate group values into individual ones is addressed by cooperative game theory. In their 1994 paper, van den Brink and Borm therefore proposed to use the Shapley (1953) value-along with the Banzhaf (1965) power index, the most prominent member of the important class of cooperative solution concepts called semi-values - to quantify node centrality as a function of the marginal contributions of a node to the centrality of groups of nodes (van den Brink \& Gilles, 2000; van den Brink \& Borm, 2002). This approach, which also had a precursor in the work by Grofman and Owen (1982), is now generally known as game-theoretic network centrality. In the wake of these pioneering papers, game-theoretic centrality has gained traction and a number of centrality measures involving various semivalues and other point (single-valued) solution concepts have been proposed in the literature (Gómez et al., 2003; Amer et al., 2012; Szczepański et al., 2012; Michalak et al., 2013b; Szczepański et al., 2016). 
A key element of the game-theoretic approach to centrality is thus to treat nodes in a graph as players in a cooperative game. A group centrality measure defines this cooperative game, and applying a single-valued solution concept then yields the game-theoretic centrality of the individual nodes. Generalising, we arrive at a generic concept of a game-theoretic centrality measure as a pair consisting of a group centrality measure and a single-valued solution concept.

In this paper, we are concerned with the computational analysis of game-theoretic centralities, and we concentrate on those that are built on semivalues. A straightforward method to compute a game-theoretic centrality measure is to successively compute the group centrality measure for each group, and then the semivalue. There are some obvious computational issues associated with this successive algorithm (which is also the optimal algorithm for certain game-theoretic centralities). Since the number of groups in a network is exponential in the size of the graph, the cooperative game that the group centrality measure defines is exponential in the size of the network (if represented extensively). Hence, computing semivalues requires an exponential number of steps. Computing the Shapley value is typically \#P-complete (Deng \& Papadimitriou, 1994; Chalkiadakis, Elkind, \& Wooldridge, 2012).

It is therefore surprising that recent research has shown that there are instances of game-theoretic centrality measures that can be computed in polynomial time, i.e., without the need to enumerate the exponential number of all possible coalitions (see Section 2 for more detail). However, existing positive computational results concern only a small number of centrality measures and each of the polynomial algorithms for these game-theoretic network centrality required an individual approach.

Against this background, the aim of this paper is to provide a broad and systematic study of which group centrality measures allow for polynomial computation of semivalues. To this end, we unify the approach to computing many of the game-theoretic network centrality measures developed to date, and many others. This will provide researchers with a convenient method for building and computing new, readily applicable centrality measures. This is especially significant given that there is no wide consensus as to which game-theoretical centrality measures are appropriate in which circumstances.

\subsection{Contributions and Organisation}

In more detail, the contributions of our paper can be summarised as follows:

- Whereas the focus of most previous work has been the computation of a single solution concept for a single group centrality, we present a generic framework for defining group centralities. Here we rely on the conception of a game-theoretic network centrality as a pair of a group centrality measure and a cooperative single-valued solution concept and exploit the structural relationships that exist between the two. If the group centrality is defined within our framework, using the conventions that we introduce, the general semivalue algorithm developed in our paper can be readily used in a plug-and-play fashion;

- By using our framework, it is possible to determine whether any semivalue of a group centrality measure can be computed in polynomial time. We show that this also facilitates the complexity analysis and the development of algorithms for classes of group centrality measures (e.g., parametrised measures) at once, rather than analysing every measure individually;

- We leverage our results to analyse the computational complexity of classes of game-theoretic measures based on the semivalue of various notions of group degree, closeness, and between- 
ness. In addition, we present the first game-theoretic centrality measures, along with their polynomial-time algorithms, for weighted and normalised degree centrality, impact factor centrality, distance-scaled and normalised betweenness centrality, and normalised closeness centrality. Although similar game-theoretic centrality measures have been proposed, the particular variation of degree, betweenness or closeness appears to have been chosen arbitrarily. Our framework facilitates the systematic study and principled comparison of many gametheoretic measures based on the many variations of these types of measures;

- We show that our framework can also be used to study game-theoretic centrality measures based on specific semivalues. Moreover, the resulting algorithms may have an even lower complexity than our general semivalue algorithm. We demonstrate the merits of this technique by developing the first polynomial-time algorithms that compute the game-theoretic centrality measures based on the Banzhaf index of power of various group degree, betweenness and closeness centrality measures.

In what follows, we discuss related work, introduce the key concepts from cooperative game theory and graph theory that are used in the paper. In Section 4 we present our framework for developing game-theoretic network centrality measures based on semivalues, and in Section 5 we show when these centrality measures can be computed in polynomial time and present an algorithm for doing so. The subsequent section presents a more focused analysis and algorithms for computing semivalues in particular of group parameterised degree, betweenness and closeness centralities. Section 7 presents a specific analysis for the Banzhaf index (as opposed to a general semivalue one) with faster (yet less general) algorithms when compared to those from the previous section. Finally, we present our conclusions and avenues for future work. The appendices follow, where we present a summary of the main notation in the paper, algorithms from Section 6 and proofs for Section 7.

\section{Related Work}

We identify two separate lines of research in game-theoretic network centrality. The first (Gómez et al., 2003; Amer \& Giménez, 2004; Skibski, Michalak, Rahwan, \& Wooldridge, 2014) is primarily concerned with applying existing and developing new cooperative single-valued solution concepts that essentially depend on an underlying network structure. Usually, the cooperative game/group centrality that these solution concepts should be applied to is not explicitly mentioned. Although the Myerson (1977) value is probably the most important such concept, other approaches have also been proposed (Amer \& Giménez, 2004).

By contrast the second line of research is concerned with applying cooperative single-valued solution concepts for general cooperative games to specific classes of cooperative games defined on networks through group centrality measures (van den Brink \& Gilles, 2000; Suri \& Narahari, 2010; Szczepański et al., 2012; Michalak, Rahwan, Szczepański, Skibski, Narayanam, Wooldridge, \& Jennings, 2013a; Szczepański, Michalak, \& Wooldridge, 2014; Szczepański, Tarkowski, Michalak, Harrenstein, \& Wooldridge, 2015; Szczepański et al., 2016). Our work contributes to this second line of research.

Game-theoretic approaches to network centrality from both lines of research have been considered for a variety of applications, such as social and organisational network analysis (Suri \& 
Narahari, 2010), biological networks (Kotter, Reid, Krumnack, Wanke, \& Sporns, 2007), terrorist networks (Lindelauf, Hamers, \& Husslage, 2013), and covert networks (Michalak et al., 2013a). ${ }^{1}$

Grofman and Owen (1982) were the first to use a cooperative single-valued solution conceptthe Banzhaf index-as a centrality measure. In their approach, a node $v$ gained value from lying on non-cyclical paths, such that-were $v$ to disappear-the other nodes in the path could not by themselves reconnect the source and destination nodes. Van den Brink and Gilles (2000) introduced the current approach to this line of research: they defined a game on the network that is based on its topology (a version of group out-degree centrality) and applied the Shapley value to it. They applied this approach to analyse dominance in digraphs. Their proposed solution is that if the node $v$ is dominated by $k$ other nodes, then the reward to every node that dominates $v$ should be split equally among all dominating nodes. Thus, the ranking of each node is determined by an aggregated reward that it obtains for dominating other nodes: for every node $w$ that a node $v$ dominates, $v$ obtains reward $\frac{1}{k}$, where $k$ is the total number of nodes dominating $w$. This ranking is axiomatised in a manner similar to the Shapley value and satisfies certain fairness properties. Ramasuri and Narahari (2008) followed by proposing the Shapley value of group degree centrality as a measure for the top- $k$ nodes problem, and they used a similar approach by considering the Shapley value of a game that modeled the influence of nodes (Suri \& Narahari, 2010). The algorithms used in both cases were approximations based on Monte Carlo-style sampling. Michalak et al. (2013b) proposed, among others, a different version of Shapley value-based degree centrality and Shapley value-based closeness centrality. Interestingly, the authors were able to develop polynomial-time algorithms for these two centrality measures. Furthermore, Szczepański et al. (2012, 2016), proposed Shapley value-based betweenness and stress centralities and also developed polynomial-time algorithms for these.

Recently, Szczepański et al. (2014) have developed the first degree-based game-theoretic centrality measure which uses the Owen value (Owen, 1977) and takes into account the so-called community structure of the underlying network. The authors also generalised both the Owen value and semivalues by proposing a new solution concept that they refer to as coalitional semivalues. Tarkowski, Szczepański, Rahwan, Michalak, and Wooldridge (2016) generalised this even further and applied the configuration value to closeness centrality. This allowed the authors to develop a centrality measure that generalises the Owen value by allowing communities to overlap. Moreover, they proposed configuration semivalues, which is a broad class that generalises both the configuration value and coalitional semivalues. Both papers developed polynomial algorithms for the computation of their respectively proposed game-theoretic centrality measures.

Table 1 illustrates how our work expands the literature on efficient computation of game-theoretic centrality measures from the second line of research. We highlight in gray the game-theoretic centrality measures that can be computed using our algorithms. Our results cover all of the measures that deal with standard networks (in addition to many more), whereas those measures that require additional information about the community structure of the network are outside the scope of our analysis.

It should be mentioned that the polynomial results listed in Table 1 are relatively rare in the grand scheme of complexity analysis for game-theoretic solution concepts. In particular, many studies on the general complexity of game-theoretic solution concepts have been carried out over the past decades (Deng \& Papadimitriou, 1994; Chalkiadakis et al., 2012), and often the solution con-

1. A broader overview of the applications of game-theoretic centrality can be found at
www.game-theoretic-centrality.com. 


\begin{tabular}{|c|c|c|}
\hline Centrality Measure & Solution Concept & First Algorithm Proposed In: \\
\hline \multirow[t]{5}{*}{ Degree } & Shapley value & Michalak et al. (2013b) \\
\hline & Banzhaf Index & this paper \\
\hline & Semivalues & this paper \\
\hline & Owen value & Szczepański et al. (2014) \\
\hline & Coalitional semivalues & Szczepański et al. (2014) \\
\hline \multirow[t]{3}{*}{ Betweenness } & Shapley value & Szczepański et al. (2012, 2016) \\
\hline & Banzhaf Index & this paper \\
\hline & Semivalues & this paper \\
\hline \multirow[t]{7}{*}{ Closeness } & Shapley value & Michalak et al. (2013b) \\
\hline & Banzhaf Index & this paper \\
\hline & Semivalues & this paper \\
\hline & Owen value & Tarkowski et al. (2016) \\
\hline & Coalitional semivalues & Tarkowski et al. (2016) \\
\hline & Configuration value & Tarkowski et al. (2016) \\
\hline & Configuration semivalues & Tarkowski et al. (2016) \\
\hline
\end{tabular}

Table 1: Papers that proposed polynomial algorithms for various game-theoretic centrality measures. Those that can be expressed in our framework and computed by our algorithms (i.e., those that do not consider communities) are highlighted in gray.

cepts of most interest are computationally intractable. Such negative results are also not uncommon for cooperative games defined on networks. For instance, Michalak et al. (2013a) established that computing the Shapley value-based centrality measure for connectivity games on graphs is \#Pcomplete. Various other cooperative games on graphs that could be used as centrality measures are also challenging (Bachrach \& Rosenschein, 2009; Greco, Malizia, Palopoli, \& Scarcello, 2011; Nebel, 2011; Aziz \& de Keijzer, 2014).

Our work can also be seen as a contribution to the literature on representation of cooperative games (Deng \& Papadimitriou, 1994; Ieong \& Shoham, 2005; Wooldridge \& Dunne, 2006; Elkind, Goldberg, Goldberg, \& Wooldridge, 2009; Greco, Malizia, Palopoli, \& Scarcello, 2009). The aim of this literature is to provide concise and computationally efficient means of representing cooperative games. Since in the context of game-theoretic network centrality the cooperative game is typically represented by a graph, we are not usually concerned with concise representation. We are, however, concerned with when certain solution concepts can be computed efficiently. For this reason, we refrain from referring to our work as a method for representing cooperative games, but rather refer to it as a framework for modeling characteristic functions on graphs. The defining feature of our framework is that it is simple to identify those cooperative games that can be computed in polynomial time with respect to the size of the graph that they are based on. Moreover, it allows for the creation of a wide variety of game-theoretic network centrality measures that - when following a set of simple guidelines-guarantees polynomial computation. 


\section{Preliminaries}

In this section, we will introduce the key concepts necessary for the understanding of the paper pertaining to cooperative game theory and graph theory. Throughout the paper we will assume acquaintance with the basic concepts of complexity theory, probability theory, and cooperative game theory. $^{2}$

\subsection{Game-Theoretic Concepts}

A cooperative game consists of a set $I=\{1,2, \ldots, n\}$ of $n$ players and a characteristic function $\nu: 2^{I} \rightarrow \mathbb{R}$, which assigns to each coalition $C \subseteq I$ of players a real value (or payoff) indicating its performance, where $\nu(\emptyset)=0$. By the grand coalition we understand the set $I$ of all players. A cooperative game in characteristic function form is a pair $(I, \nu)$, but to avoid cluttered notation we will simply refer to it by $\nu$. In our network setting we will always consider the set of players to be the set of nodes $V$ in a graph.

A basic research problem in cooperative game theory is how to divide the payoff from cooperation among the players. Semivalues (Dubey, Neyman, \& Weber, 1981) represent an important class of solutions to this problem. The key assumption of this class of solution concepts is that they determine the payoff of a player based on this player's contribution to each group. In simplest terms, semivalues assign to each player a weighted average of that player's marginal contributions. The defining characteristic of semivalues is that the weights of marginal contributions of a player to groups of the same size have to be the same. Semivalues can thus be interpreted as the expected contribution that a player will make to a random group.

To make this formally precise, we denote by $\operatorname{MC}(C, i)$ the marginal contribution of the player $i$ to the coalition $C \cup\{i\}$, i.e., $\operatorname{MC}(C, i)=\nu(C \cup\{i\})-\nu(C)$. We let $\beta:\{0,1, \ldots, n-1\} \rightarrow$ $[0,1]$ be a function such that $\sum_{k=0}^{n-1} \beta(k)=1$. Accordingly, $\beta(k)$ represents the probability that a player contributes to a coalition of size $k$. Although — in principle - this can be any function, we assume that it can be computed in polynomial time. Given $\beta$, the semivalue $\phi_{i}(\nu)$ for a player $i$ in cooperative game $\nu$ is now defined as:

$$
\phi_{i}(\nu)=\sum_{0 \leq k<n} \beta(k) \mathbb{E}\left[\mathrm{MC}\left(C_{k}, i\right)\right]
$$

where $C_{k}$ is the random variable of all possible coalitions of size $k$ drawn with uniform probability form the set $I \backslash\{i\}$, and $\mathbb{E}[\cdot]$ is the expected value operator.

The Shapley (1953) value and the Banzhaf (1965) index of power are two prominent and wellknown examples of semivalues. They are defined by $\beta$-functions $\beta^{\text {Shapley }}$ and $\beta^{\text {Banzhaf }}$, given by, respectively:

$$
\beta^{\text {Shapley }}(k)=\frac{1}{n} \quad \quad \beta^{\text {Banzhaf }}(k)=\frac{\left(\begin{array}{c}
n-1 \\
k
\end{array}\right)}{2^{n-1}} .
$$

In other words, the Shapley value assumes that given the player set $I$ and a coalition $C$ of size $k$, the weight of the marginal contribution of any player $i \in I \backslash C$ to $C$ is $\frac{1}{k\left(\begin{array}{c}n \\ k\end{array}\right)}$ (i.e., there is a uniform probability distribution over the set of sizes of coalitions). Conversely, the Banzhaf index of

2. We refer the reader to the books by Papadimitriou (2003), Tijms (2012), and Maschler, Solan, and Zamir (2013) for excellent introductions to these fields. 
power gives every coalition (rather than the size of the coalition) the same weight, i.e., $\frac{1}{2^{n}}$. This is equivalent to saying that the probability of a coalition of size $k$ forming is $\frac{\left(\begin{array}{c}n-1 \\ k\end{array}\right)}{2^{n-1}}$.

The significance of semivalues for our work is that they provide a method for specifying the importance of certain types of synergy in a given context. A trivial example is to give a weight of 1 to the empty coalition (i.e., $\beta(0)=1$ ) and 0 to all other coalitions. In this case, synergy is not considered at all. If this semivalue is applied to group degree, group betweenness or group closeness, then the resulting measure is the classic degree, betweenness or closeness measure. In essence, this answers the question "what can a player achieve on his or her own." Going further, by varying the ratio between the weight of the empty coalition and the non-empty coalitions, it is possible to specify the relative impact of synergy on the ranking of players. On the other hand, giving coalitions of size $n-1$ a weight of 1 (i.e., $\beta(n-1)=1$ ) and all other coalitions a weight of 0 , the resulting semivalue answers the question "how much would all the players lose were a single player to leave." Semivalues offer the possibility of fine-tuning the types of contributions and synergies that are important in determining the worth of players or-in the case of networks-nodes.

\subsection{Graph-Theoretic Concepts}

A network is a weighted digraph $D=(V, E, \omega)$, where $V$ is a set of nodes, $E$ is a set of edges, i.e., ordered pairs $(v, u)$ of nodes in $V$ with $v \neq u$, and $\omega: E \rightarrow \mathbb{R}^{+}$is a weight function from edges to the positive real numbers. A weighted digraph $D=(V, E, \omega)$ is said to be undirected if (i) $(v, u) \in E$ implies $(u, v) \in E$ and (ii) $\omega((v, u))=\omega((u, v))$ for all $(u, v) \in E$. In that case, $D$ is also said to be a weighted graph. A weighted graph or digraph $D=(V, E, \omega)$ is said to be unweighted if $\omega(e)=1$ for all $e \in E$. Unweighted graphs and unweighted digraphs we also refer to as simply graphs and digraphs, respectively. In that case, we will generally omit the reference to $\omega$ in the signature.

A path $\pi_{s t}$ from a source node $s$ to destination node $t$ in a graph $G$ is an ordered set $\left(v_{0}, v_{1}, \ldots, v_{k}\right)$ such that $v_{0}=s$ and $v_{k}=t$ and $\left(v_{i}, v_{i+1}\right) \in E$ for all $i$ with $1 \leq i<k$. The length of a path $\pi_{s t}$ is the sum of the weights of the edges that it is comprised of. We will define Paths $(s, t)$ as the set of shortest paths between nodes $s$ and $t$. We define the set of neighbours of a node $v$ by $E(i)=\{j$ : $(i, j) \in E\}$. The neighbour set of a subset $C$ of nodes is defined as $E(C)=\bigcup_{i \in C} E(i) \backslash C$. We refer to the degree of a node $v$ in a graph by $\operatorname{deg}(v)=|E(v)| .{ }^{3}$ The distance from a node $s$ to a node $t$ is denoted by $\operatorname{dist}(s, t)$ and is defined as the length of the shortest path between $s$ and $t$. The distance between a node $v$ and a subset of nodes $C \subseteq V$ is denoted by $\operatorname{dist}(C, v)=\min _{u \in C} \operatorname{dist}(u, v)$. Paths, neighbours, and distances are prominent in definitions of network centrality measures, i.e., functions that associate with each node a real value that represents its centrality, and we will refer to them as (graph) items.

\subsubsection{Classic Node Centrality}

We now present the three classic notions of node centrality as introduced by Freeman (1979).

1. Degree Centrality ranks nodes according to how many neighbours they have. The larger the neighbourhood of a node, the more central it is. Formally:

$$
\operatorname{degree}(v)=|E(v)|
$$

3. This is referred to as the out-degree in weighted graphs. 
2. Betweenness Centrality ranks a node according to how many shortest paths in the network it lies on. Often the additional constraint is added that paths do not contribute to the rank of their source and destination nodes. Formally, let $\sigma_{(s, t)}=\mid$ Paths $(s, t) \mid$, or the number of shortest paths between $s$ and $t$, and $\sigma_{(s, t)}(v)$ be the number of shortest paths between $s$ and $t$ that $v$ lies on, with the assumption that $s \neq v$ and $t \neq v$ (otherwise $\sigma_{(s, t)}(v)=0$, although this assumption is not always upheld). Given this, betweenness centrality is defined as follows:

$$
\operatorname{betweenness}(v)=\sum_{s \neq v} \sum_{t \neq s, v} \frac{\sigma_{(s, t)}(v)}{\sigma_{(s, t)}}
$$

3. Closeness Centrality ranks nodes based on their distances to other nodes. The most classic version of the centrality ranks a node according to the sum of the distances from it to any other node in the network:

$$
\operatorname{closeness}(v)=\sum_{u \in V} \operatorname{dist}(v, u)
$$

This results in an inverse ranking (i.e., nodes that are more important have smaller value). A variation on closeness - the influence game - was introduced by Michalak et al. (2013b). This centrality results in a ranking such that more important nodes have higher value by using some non-increasing function of distance, $f: \mathbb{R} \rightarrow \mathbb{R}$, rather than distance itself. Thus, influence games are defined as follows:

$$
\operatorname{influence}_{-} \operatorname{game}(v)=\sum_{u \in V} f(\operatorname{dist}(v, u)) .
$$

When

$$
f(\operatorname{dist}(v, u))=\frac{1}{\operatorname{dist}(v, u)},
$$

with the assumption that $f(\operatorname{dist}(v, u))=0$ if there is no path between $v$ and $u$, then the resulting measure is known as harmonic closeness (Boldi \& Vigna, 2014).

\subsubsection{Group CENTRALity}

We present below some classic group centralities due to Everett and Borgatti (1999). ${ }^{4}$ These group centrality measures are a natural extension of the centrality measures introduced by Freeman (1979).

1. Group Degree Centrality of a group of nodes is defined as the size of the neighbourhood of this group. Formally, group degree centrality in a graph $G$ is defined as:

$$
\psi^{D}(G)(S)=|\{v: v \in E(S)\}| .
$$

2. Group Betweenness Centrality of a group of nodes $S$ is defined as the number of shortest paths that visit at least one node in $S$. Often (but not always), the additional assumption is made that the source and destination nodes of these paths cannot belong to $S$. Let $\sigma_{(s, t)}=|\operatorname{Paths}(s, t)|$ and $\sigma_{(s, t)}(S)$ be the number of shortest paths between $s$ and $t$ that visit at least one node

4. In Section 6 we will also consider more general versions of these measures. 


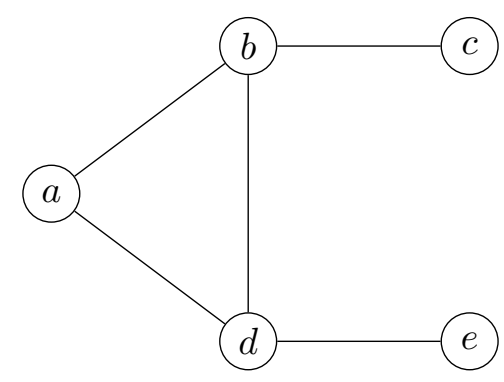

Figure 1: A sample unweighted network of 5 nodes.

in $S$, with the assumption that $s \notin S$ and $t \notin S$ (otherwise $\sigma_{(s, t)}(S)=0$ ). Formally, group betweenness centrality in a graph $G$ is defined as:

$$
\psi^{B}(G)(S)=\nu_{G}^{B}(S)=\sum_{s, t \notin S} \frac{\sigma_{(s, t)}(S)}{\sigma_{(s, t)}} .
$$

3. Group Closeness Centrality of a group of nodes $S$ is defined as the sum of the distances from $S$, to any node outside of $S$. This results in a ranking, where groups with lower value are - on average — closer to the nodes outside of the group, and therefore more central. Formally, group closeness centrality in a graph $G$ is defined as:

$$
\psi^{C L}(G)(S)=\nu_{G}^{C L}(S)=\sum_{v \in V} \operatorname{dist}(S, v)
$$

The influence game introduced previously for single nodes was actually defined by Michalak et al. (2013b) as a group centrality measure. It is defined with the help of a non-increasing function $f: \mathbb{R} \rightarrow \mathbb{R}$ in the following manner:

$$
\psi_{f}^{C L}(G)(S)=\nu_{G, f}^{C L}(S)=\sum_{v \in V} f(\operatorname{dist}(S, v)) .
$$

When $f(k)=\frac{1}{k}$, we call the resulting measure harmonic group closeness (Boldi \& Vigna, 2014).

Definition 1 (Group centrality) In general, we will use the variable $\psi$ to denote a group centrality measure. Formally, $\psi$ maps any graph $G=(V, E)$ onto a characteristic function $\nu: 2^{V} \rightarrow \mathbb{R}$.

\subsubsection{Game-Theoretic CENTRALity}

We are now in a position to define game-theoretic centrality, which can be viewed as a method to rank nodes by aggregating the centralities of (typically all) groups of nodes in a network. In particular, in this approach nodes are treated as players in a cooperative game and their groups as coalitions. ${ }^{5}$ The characteristic function is typically some group centrality measure (such as those described above) and a chosen single-valued solution concept is the method of aggregation.

5. The centrality of edges can also be of interest (Girvan \& Newman, 2002), however in this paper we are focused on ranking nodes. 


\begin{tabular}{cccccccc}
\hline$C$ & $\nu^{B}(C)$ & $C$ & $\nu^{B}(C)$ & $C$ & $\nu^{B}(C)$ & $C$ & $\nu^{B}(C)$ \\
\hline$\emptyset$ & 0 & $\{a, b\}$ & 4 & $\{a, b, c\}$ & 0 & $\{a, b, c, d\}$ & 0 \\
$\{a\}$ & 0 & $\{a, c\}$ & 0 & $\{a, b, d\}$ & 2 & $\{a, b, c, e\}$ & 0 \\
$\{b\}$ & 6 & $\{a, d\}$ & 4 & $\{a, b, e\}$ & 2 & $\{a, b, d, e\}$ & 0 \\
$\{c\}$ & 0 & $\{a, e\}$ & 0 & $\{a, c, d\}$ & 2 & $\{a, c, d, e\}$ & 0 \\
$\{d\}$ & 6 & $\{b, c\}$ & 0 & $\{a, c, e\}$ & 0 & $\{b, c, d, e\}$ & 0 \\
$\{e\}$ & 0 & $\{b, d\}$ & 6 & $\{a, d, e\}$ & 0 & $\{a, b, c, d, e\}$ & 0 \\
& & $\{b, e\}$ & 4 & $\{b, c, d\}$ & 2 & & \\
& & $\{c, d\}$ & 4 & $\{b, c, e\}$ & 0 & & \\
& & $\{c, e\}$ & 0 & $\{b, d, e\}$ & 2 & & \\
& $\{d, e\}$ & 0 & $\{c, d, e\}$ & 0 & & \\
\hline
\end{tabular}

Table 2: The betweenness centralities of the coalitions of the network in Figure 1.

In the context of this paper, a pair of any given group centrality measure and single-valued solution concept can be considered a centrality measure, since it yields a ranking of nodes in any graph.

Definition 2 (Game-theoretic centrality measure) Formally, we define a game-theoretic centrality measure as a pair $(\psi, \phi)$ consisting of a group centrality $\psi$ and a cooperative single-valued solution concept $\phi$.

Example 1 Let us consider the game-theoretic centrality measure $\left(\psi^{B}, \phi^{\text {Shapley }}\right)$. For a specific graph $G=(V, E)$, the importance of each node $u \in V$ according to this measure is evaluated by the Shapley value of the game $\psi^{B}(G)$, i.e., $\phi_{u}^{\text {Shapley }}\left(\psi^{B}(G)\right)$. Since we started off with a group centrality measure called group betweenness centrality and the Shapley value to it, we call the resulting centrality measure Shapley betweenness centrality.

Let us now look at an example to illustrate the concepts discussed thus far.

Example 2 Consider betweenness centrality and group betweenness centrality in the unweighted directed network $G$ depicted in Figure 1. Observe that, besides the direct edges, there are also the following shortest paths between nodes:

$$
a b c, a d e, b d e, c b a, c b d, c b d e, d b c, e d a, e d b, e d b c .
$$

It can then easily be established that the betweenness centrality for nodes $a, c$, and $e$ is 0 , whereas that of $b$ and $d$ totals to 6 . The group centralities $\nu^{B}(C)$ (omitting the reference to $G$ ) of the groups of nodes (coalitions) $C$ are summarised in Table 2. We can now use the Shapley value to aggregate the group centralities for the individual nodes. In this way, we find that the game-theoretical betweenness centralities for the Shapley value of the nodes $a, b, c, d$, and e are, respectively, $\frac{-2}{5}, \frac{3}{10}$, $\frac{-7}{6}, \frac{3}{10}$, and $\frac{-7}{6}$.

In the next section we will introduce our framework for defining group centrality measures. We will later show that with some simple restrictions, when the group centrality measures defined in our framework are paired with semivalues, then the resulting game-theoretic measure can be computed in polynomial time. However, before we move on, let us illustrate the differences between the three approaches to centrality that we mentioned via an example. 


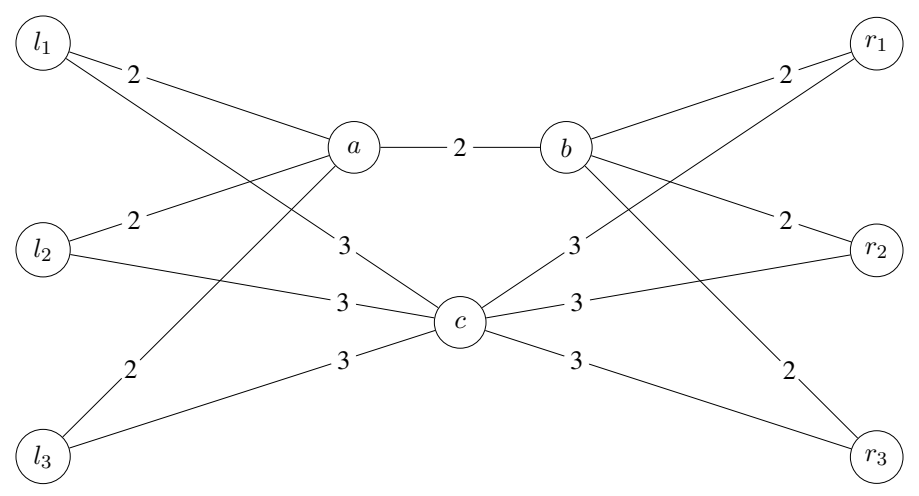

Figure 2: A graph representing a delivery network. Edge weights represent the physical distance between nodes.

Example 3 Consider a delivery network, where parcels (for instance of data or physical mail) are transferred between nodes. Figure 2 presents an example of such a network. Edge weights represent the distance between nodes. Assume that we want to intercept parcels travelling through this network, while the only thing we know is that deliveries are always made via the shortest route. We can try to achieve this by placing a "mole" at any of the nodes. For this task, we can model the importance of a node $v$ using a variant of betweenness centrality that we call stress centrality after Szczepański et al. $(2012,2016):^{6}$

$$
\operatorname{stress}(v)=\sum_{s \in V} \sum_{t \in V} \sigma_{(s, t)}(v)
$$

If we can only place one mole in this setting, then this "classic" approach to betweenness centrality would give us the correct answer: nodes $a$ and $b$ are the best nodes to place the mole, since they control the flow of 44 different shortest paths each, which is more than any other node. However, what if we can place two moles? Unfortunately, the classic approach to centrality is of little help here, since placing moles at the two nodes with the highest stress centrality- $a$ and $b$ does not guarantee us the greatest success. We could, for instance, do better by placing a mole on $b$ and another one on $c$, as together they control a larger number of delivery paths. For this analysis, we must consider the group stress centrality, which is defined for any group of nodes $S$ as follows:

$$
\operatorname{group} \_ \text {stress }(S)=\sum_{s \in V} \sum_{t \in V} \sigma_{(s, t)}(S)
$$

We can now see that whereas the group $\{a, b\}$ controls a combined number of 56 shortest paths, the two optimal groups of size $2-\{a, c\}$ and $\{b, c\}-$ control 80 shortest paths each. Although the distinction between the node centrality and group centrality in this case seems trivial given that only the domain changes between the two definitions, two decades separate the two areas of research.

On the other hand, what would be the best approach to defend against the random placement of moles? Assume our objective is to secure as many of the shortest paths as possible, i.e., clearing

6. Since moles can also affect parcels at their own location, we make the slight modification of also counting towards a node's centrality the shortest paths that originate and end at this node. 
them from all moles that may be on them. Also assume that we can send a single team to a single node to investigate whether it has been compromised, and, if so, to neutralise the resident mole. Which node should we send the team to first? This depends strongly on the number of nodes that we believe are compromised. The catch is that by neutralising a mole at a node, we may not succeed in securing any shortest paths through it, as each of them may also be compromised by other moles. Consider the case where we know that two moles are compromised (although any probability distribution that models how many moles are likely to exist in the network can be used).

The question of where to send the team first is a complex issue and it is not immediately obvious what we should optimise for. For instance, there is no "quickest" way to ensure that all paths are not compromised. Given that the placement of moles is random, this would require the checking of all nodes. One criterion that we can optimise for is to ensure that we clear as many shortest paths as possible with our first visit. Intuitively, this would mean that we should visit a or b first, since they control the most paths. This, however, is not the case given the following two considerations:

1. The shortest paths that a lies on contain more nodes on average than those that clies on. We note that it is more likely that shortest paths that contain more nodes are compromised, since a mole at any one node on the path would suffice to tamper with deliveries. Given this, if we know that there are two moles, then the likelihood of the second mole lying on a large number of the same shortest paths that a lies on is higher than for $c$. This means that even if we clear a of moles, the likelihood that the shortest paths it lies on are still compromised is higher than for $c$.

2. Node a lies on many of the same shortest paths as $b$. This means that if $b$ is the other infected node, then clearing a of a mole leaves most of its paths still compromised. However-given that c has few common shortest paths with other nodes-clearing c of a mole will clear most of its shortest paths with absolute certainty.

As an example, consider all shortest paths from some $l_{i}$ to some $r_{j}$ for $i, j \in\{1,2,3\}$. There are 9 paths of the form $\left(l_{i}, a, b, r_{j}\right)$ and 9 of the form $\left(l_{i}, c, r_{j}\right)$. We see both considerations at work here:

1. The paths of this form that a lies on contain 4 nodes, whereas those that c lies on contain 3 nodes, and

2. All 9 of the paths of this form that a lies on overlap with those that b lies on. This means that if $b$ also has a mole, then clearing a will leave all of these paths compromised. On the other hand, if c has a mole, then clearing it guarantees that at least 6 paths of this form are not compromised.

In order to capture the complex interactions between nodes and the synergies (both positive and negative) that nodes exhibit when considered in groups, the semivalue approach needs to be considered in order to analyse the expected number of shortest paths "sterilised" after clearing a node. The semivalue for group stress centrality of a node $v$ with the $\beta$ function such that $\beta(k)=1$ for $k=1$ and $\beta(k)=0$ otherwise gives us exactly the number of shortest paths that we will cure upon removing a mole at a given location, given that all other nodes are equally likely to be compromised. If $c$ is compromised, then removing a mole placed there will cure an expected number of $24 \frac{3}{4}$ shortest paths, whereas removing a mole at a or $b$ will cure only an expected number of $16 \frac{3}{4}$ shortest paths. 


\section{A Generic Framework for Game-Theoretic Network Centrality}

We will now formally introduce our generic framework for modeling group centrality measures (i.e., characteristic functions on graphs). We will refer to the class of group centrality measures that can be expressed by using this framework as $\mathscr{M}$, which is an intermediary step in our ultimate goal of finding within $\mathscr{M}$ those group centralities that allow for polynomial computation of semivalues. $\mathscr{M}$ will allow us to abstract away the various types of considerations that make nodes central (i.e., shortest paths, neighbours, or closeness to other nodes). ${ }^{7}$ The remainder of this section will be dedicated to introducing our framework, which consists of two main components: the first we call items, and the second relations. In the two following subsections we will introduce both components, and in the third we will show how they are combined to model characteristic functions (i.e., group centrality measures) on graphs.

\subsection{Items}

Items refer to the graph-theoretic entities that influence the centrality of groups of nodes given a particular group centrality measure. For instance, for group betweenness centrality, the items that influence centrality are the shortest paths in the network. In the case of group degree centrality, items are simply nodes, since centrality is derived from having neighbours. If a node neighours a group, then it contributes to the group's centrality. In the case of group closeness centrality, nodes impact centrality like in degree centrality, but the magnitude of their impact on the ranking is based on their distance from the group rather than the number of them that neighbour it.

Formally, we assume that the characteristic function $\nu_{G}$ (as given by some group centrality measure, i.e., $\nu_{G}=\psi(G)$ ) can be computed by counting these items. We will typically denote a single item by $\vartheta$. Each item $\vartheta$ has its own real value, denoted by $f(\vartheta) \in \mathbb{R}$, and it is this value that impacts the value of nodes and coalitions. Given a group centrality measure, the set of all items in a graph $G$ is denoted by $\Theta_{G}$. We will also partition $\Theta_{G}$ into pairwise disjoint groups $\Theta_{1}, \Theta_{2}, \ldots, \Theta_{l}, \ldots, \Theta_{h(G)}$ of items according to their value. That is, items from the same group should have the same value, whereas the value of any two items from different groups should be different. We will denote the size of the partition, which depends on the graph in question and the group centrality measure under consideration, by $h(G)$. This value will be central to the complexity analysis of our algorithms. Later we will see how such partitions facilitate the computation of semivalues. In particular, even in the presence of an exponential number of items (such as in the case of betweenness), the size, $h(G)$, of such a partition may be polynomial.

\subsection{Relations}

Relations are the second component of our framework and control how items affect the value of nodes and coalitions (in effect, defining a characteristic function). In particular, we define three types of relations: positive, negative and neutral. In simplest terms, the value of a coalition will be the sum of the values of items that it is positively related to. Deciding which items are positively related to the coalition, however, depends on the individual relations of the nodes to these items. For example, in the context of group degree centrality, nodes are positively related to their neighbours, negatively related to themselves and neutrally related to all other nodes in the network. These three

7. Next, in Section 5 we are able to put just a few restrictions on our abstract framework such that polynomial computation is guaranteed, resulting in a subclass of $\mathscr{M}$ that can be computed in polynomial time. 
relations are pairwise mutually exclusive and otherwise independent of one another. We will later see how we can use them to determine the marginal contributions of nodes, and-in effect-compute semivalues. For individual nodes, the relations are as follows:

$N$ : is the neutral relation. $N(u, \vartheta)$ simply indicates that the node $u$ and item $\vartheta$ are unrelated, and $u$ does not impact the value of any coalition through $\vartheta$.

$R$ : is the positive relation. $R(u, \vartheta)$ indicates that $u$ is positively related to $\vartheta$, meaning that $u$ contributes the value $f(\vartheta)$ when joining a coalition $C$ as long as all nodes in $C$ are neutrally related to $\vartheta$.

$\widetilde{R}$ : is the negative relation. $\widetilde{R}(u, \vartheta)$ indicates that $u$ is negatively related to $\vartheta$, meaning that $u$ subtracts the value $f(\vartheta)$ when joining a coalition $C$ if it has already been contributed by some node in $C$ but not yet taken away by another node in $C$ that is negatively related to $\vartheta$.

Now that we have introduced relations between nodes and items, we can formally extend them to relations between groups of nodes/coalitions and items.

$$
\begin{aligned}
& N(C, \vartheta) \text { if and only if } \quad \forall_{v \in C} N(v, \vartheta), \\
& R(C, \vartheta) \text { if and only if } \quad \exists_{v \in C} R(v, \vartheta) \text { and } \neg \exists_{u \in C} \widetilde{R}(u, \vartheta), \\
& \widetilde{R}(C, \vartheta) \text { if and only if } \quad \exists_{v \in C} \widetilde{R}(v, \vartheta) .
\end{aligned}
$$

Observe that the relations as defined for coalitions are also pairwise mutually exclusive. Intuitively, this means that if $\widetilde{R}(C, \vartheta)$ holds, then some node belongs to $C$ that precludes $C$ from ever benefiting from the item $\vartheta$, even when other nodes join the coalition. $R(C, \vartheta)$, on the other hand, means that $f(\vartheta)$ (the value of $\vartheta$ ) is counted towards the value of $C$. As mentioned previously, the value of a coalition is the sum of the values of the items to which it is positively related. In this case, if a node $u$ such that $\widetilde{R}(u, \vartheta)$ ever joins $C$, then it will subtract $f(\vartheta)$ from the value of the coalition. On the other, $N(C, \vartheta)$ means that $f(\vartheta)$ can still be contributed by any node $u$ for which $R(u, \vartheta)$ holds.

Example 4 Consider again the network in Example 2, in particular the characteristic function $\nu^{B}$ that represents the betweenness group centralities. This characteristic function/group centrality we can now model by choosing appropriate items, relations, and the function $f$. For each coalition $C$, we can then express $\nu^{B}(C)$ as a function of these. As the set of items we choose the set of shortest paths $\pi_{s t}$ between any two distinct nodes $s$ and $t$. For each item, i.e., shortest path $\pi_{s t}$, we set $f\left(\pi_{s t}\right)=1$. We say that node $x$ is positively related to shortest path $\pi_{s t}-o r$, in our symbolism, $R\left(\pi_{s t}, x\right)$-if $\pi_{s t}$ visits $x$ and $x \neq s, t$. Moreover, $\widetilde{R}\left(\pi_{s t}, x\right)$ if $x=s$ or $x=t$, and $N\left(\pi_{s t}, x\right)$ if neither $R\left(\pi_{s t}, x\right)$ nor $\widetilde{R}\left(\pi_{s t}, x\right)$.

In the example, we see that, for instance, node $b$ is positively related to the paths

$$
a b c, c b a, c b d, c b d e, d b c, e d b c
$$

This reflects b's betweenness centrality of 6. Similarly, node d is positively related to

$$
\text { ade, bde, } c b d e, e d a, e d b, e d b c
$$




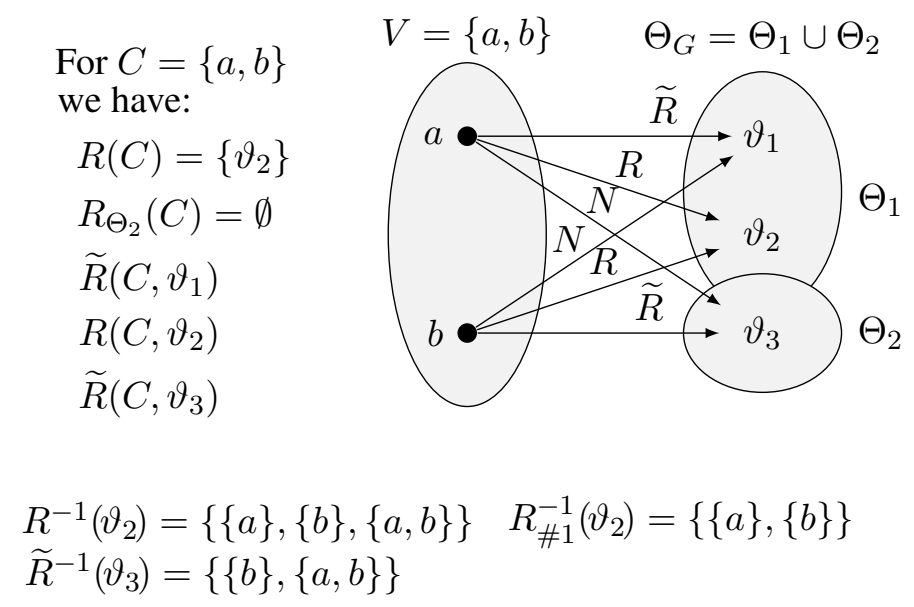

Figure 3: An example of notation used to describe relations between nodes and items.

signifying that the betweenness centrality of $d$ is likewise 6 . As $b$ and $d$ are negatively related to a path if they occur as one of the endpoints, we find that the coalition $\{b, d\}$ is positively related to the paths

$$
a b c, a d e, c b a, c b d e, e d a, e d b c .
$$

We thus find that $\nu^{B}(\{b, d\})$ equals

$$
\sum_{\vartheta \in R(\{b, d\})} f(\vartheta)=f(a b c)+f(a d e)+f(c b a)+f(c b d e)+f(e d a)+f(e d b c)=6 .
$$

In the next section we will generalise this idea. Observe that node a is negatively related to abc, ade, $c b a$, eda. Hence, if a were to join $\{b, d\}$, we find that $\sum_{\vartheta \in R(\{a, b, d\})} f(\vartheta)=f(c b d e)+f(e d b c)=2$, which neatly coincides with $\nu^{B}(\{a, b, d\})$.

For clarity, we will use the following notation for the relation $R$ (and similarly for $\widetilde{R}$ and $N$ ). This notation will allow us to express the class $\mathscr{M}$ formally and concisely.

$$
\begin{aligned}
R(C) & =\left\{\vartheta \in \Theta_{G}: R(C, \vartheta)\right\} & & R_{\Theta_{l}}(C)=\left\{\vartheta \in \Theta_{l}: R(C, \vartheta)\right\}, \\
R^{-1}(\vartheta) & =\{C \subseteq V: R(C, \vartheta)\} & & R_{\# k}^{-1}(\vartheta)=\{C \subseteq V: R(C, \vartheta) \text { and }|C|=k\} .
\end{aligned}
$$

Thus, $R(C)$ denotes the set of items positively related to the coalition $C, R_{\Theta_{l}}(C)$ the set of items in group $\Theta_{l}$ that is positively related to $C, R^{-1}(\vartheta)$ the set of coalitions to which item $\vartheta$ is positively related, and $R_{\# k}^{-1}(\vartheta)$ the set of coalitions of size $k$ to which $\vartheta$ is positively related. Note that in the case of a singleton coalition we write $R(u)$ instead of $R(\{u\})$ to avoid cluttered notation.

\subsection{Model of a Characteristic Function}

We are now in a position to combine all of the elements of our framework and show how they can be used to model characteristic functions on graphs. 
Definition 3 (Model of a characteristic function) Given a graph $G=(V, E)$ and a characteristic function $\nu: 2^{V} \rightarrow \mathbb{R}$, a model of $\nu$ is a tuple

$$
M_{G}=\left(\Theta_{G},\left\{\Theta_{1}, \ldots, \Theta_{h(G)}\right\}, R, \widetilde{R}, N, f, g\right),
$$

where $\left\{\Theta_{1}, \ldots, \Theta_{h(G)}\right\}$ is a partition of a set $\Theta_{G}$ of items, $R, \widetilde{R}$ and $N$ are the positive, negative and neutral association relations, respectively, and $f: \Theta_{G} \rightarrow \mathbb{R}$ and $g: \mathbb{N} \rightarrow \mathbb{R}$ are real-valued functions, such that for all coalitions $C \subseteq V$ the following property holds:

$$
\nu(C)=g(|C|) \sum_{\vartheta \in R(C)} f(\vartheta) .
$$

Observe that the relation $R$ depends on $\widetilde{R}$, which plays a role in determining whether a coalition can be positively related to an item. Moreover, any pair of relations from $R, \widetilde{R}$, and $N$ can be used to determine the third, however we include all three for the sake of completeness and convenience. For the purposes of this paper, we want to group similar items together using the partition of items, and so we focus on models for which a number of structural regularity constraints hold:

$$
\begin{array}{ll}
\forall_{1 \leq l \leq h(G)} \forall_{\vartheta_{i}, \vartheta_{j} \in \Theta_{l}:} & f\left(\vartheta_{i}\right)=f\left(\vartheta_{j}\right) ; \\
\forall_{1 \leq l \leq h(G)} \forall_{\vartheta_{i}, \vartheta_{j} \in \Theta_{l}}: & \left|N_{\# k}^{-1}\left(\vartheta_{i}\right)\right|=\left|N_{\# k}^{-1}\left(\vartheta_{j}\right)\right| ; \\
\forall_{1 \leq l \leq h(G)} \forall_{\vartheta_{i}, \vartheta_{j} \in \Theta_{l}}: & \left|R_{\# k}^{-1}\left(\vartheta_{i}\right)\right|=\left|R_{\# k}^{-1}\left(\vartheta_{j}\right)\right| .
\end{array}
$$

If these properties hold and $\vartheta \in \Theta_{l}$, we also let, with a slight abuse of notation:

$$
\begin{aligned}
f\left(\Theta_{l}\right) & =f(\vartheta), & N_{\# k}^{-1}\left(\Theta_{l}\right) & =N_{\# k}^{-1}(\vartheta), \\
R_{\# k}^{-1}\left(\Theta_{l}\right) & =R_{\# k}^{-1}(\vartheta), & \widetilde{R}_{\# k}^{-1}\left(\Theta_{l}\right) & =\widetilde{R}_{\# k}^{-1}(\vartheta) .
\end{aligned}
$$

Property $(M 1)$ states that the value of a coalition is the product of a function of its size and the sum of the contributions of its members over all positively related items. Property $(M 2)$ states that the value of every item in a part must be the same. Moreover, Properties (M3), and (M4) state that the set of coalitions of size $k$ to which any item is related (by relation $R, N$, and consequently by $\widetilde{R}$ ) is the same for items in the same part of the partition.

Note that the properties $(M 2),(M 3)$ and $(M 4)$ do not limit the expressiveness of the model. Rather, they are introduced as a matter of convention in order to group similar items together in the partition of items. A partition that contains only singletons will always satisfy these properties. However, such a partition will often be prohibitively large. We will later see that graph structure from which the centrality of groups is derived imposes structure on the group centrality itself (thereby not being fully expressive) that-in some cases-reduces the computational complexity of gametheoretic solution concepts under consideration. In the following sections, our framework will allow us to study in a principled fashion how group centrality, single-valued solution concepts and classes of cooperative games that can be described by graphs interact to impact complexity.

First, however, we have to show how models of characteristic functions relate to group centrality measures, i.e., functions mapping graphs to characteristic functions. We do this by introducing the class $\mathscr{M}$ of group centrality measures:

Definition 4 (The class $\mathscr{M}$ ) $\mathscr{M}$ is the class of all group centrality measures such that for all $\psi \in$ $\mathscr{M}$, and for every graph $G$, there exists a model $M_{G}$ of $\psi(G)$ such that $M_{G}$ has the properties (M1), (M2), (M3), and (M4). 
Let us illustrate using an example how the various elements of a model interact in order to define a characteristic function.

Example 5 Let us consider Figure 3. We want to find an $M_{G}=\left(\Theta_{1}, \Theta_{2}, R, \widetilde{R}, f, g\right)$ such that it models $\nu_{G}(\{a\})=1, \nu_{G}(\{b\})=1, \nu_{G}(\{a, b\})=2$. We define $f\left(\vartheta_{1}\right)=f\left(\vartheta_{2}\right)=1, f\left(\vartheta_{3}\right)=2($ satisfying (M2) ) and $g(k)=k$. Property $(M 1)$ holds, since $\nu_{G}(\{a\})=\nu_{G}(\{b\})=g(1) f\left(\vartheta_{2}\right)$ and $\nu_{G}(\{a, b\})=g(2) f\left(\vartheta_{2}\right)$. However, property $(M 3)$ is not satisfied since $\left|N_{\# 1}^{-1}\left(\vartheta_{1}\right)\right| \neq\left|N_{\# 1}^{-1}\left(\vartheta_{2}\right)\right|$. This can be fixed by defining three groups of singletons instead. In fact, a partition of singletons will always satisfy properties (M2), (M3) and (M4). The motivation for introducing groups will become apparent in the next section, where we will show how restricting the number of groups (without restricting the number of items) will yield polynomial computation.

\section{Computational Analysis}

For any group centrality measure $\psi$ and semivalue $\phi$, we define the problem $\operatorname{Semivalue}(\psi, \phi)$ to compute the centrality of a node $u$ according to the game-theoretic centrality measure $(\psi, \phi)$ :

$\operatorname{Semivalue}(\psi, \phi)$

Given: $\quad$ Graph $G=(V, E)$, node $u \in V$

Problem: Compute $\phi_{u}(\psi(G))$

In general, this problem is intractable (Deng \& Papadimitriou, 1994; Chalkiadakis et al., 2012) and it is not immediately obvious what restrictions on the game-theoretic centrality measures would yield polynomial computation. Even if we restrict ourselves to the class of group centrality measures $\mathscr{M}$, there are still functions in this class for which $\operatorname{Semivalue}(\psi, \phi)$ is intractable (for example, this could happen if $h(G)$ is exponential in the size of $V$ ). However, we will define a subclass $\mathscr{M}^{*}$ of $\mathscr{M}$ such that $\operatorname{Semivalue}(\psi, \phi)$ can be computed in polynomial time for all $\psi \in \mathscr{M}^{*}$.

Definition 5 (The subclass $\mathscr{M}^{*}$ of $\mathscr{M}$ ) $\mathscr{M}^{*}$ is the subclass of group centrality measures in $\mathscr{M}$ for which additionally the following properties hold:

$\left(M 1^{*}\right) \exists_{n \in \mathbb{N}} \forall_{G} \exists_{M_{G}} M_{G}$ models $\psi(G)$ and $h(G) \leq O\left(|V|^{n}\right)$;

$\left(M 2^{*}\right) \exists_{n \in \mathbb{N}} \forall_{G}$ there exists a model $\left(\Theta_{G},\left\{\Theta_{1}, \ldots, \Theta_{h(G)}\right\}, R, \widetilde{R}, f, g\right)$ of $\psi(G)$ such that the function that for each $u \in V, k$, and l returns the values $f\left(\Theta_{l}\right), g(k),\left|R_{\Theta_{l}}(u)\right|,\left|\widetilde{R}_{\Theta_{l}}(u)\right|$, $\left|N_{\# k}^{-1}\left(\Theta_{l}\right)\right|$, and $\left|R_{\# k}^{-1}\left(\Theta_{l}\right)\right|$ is computable in time $O\left(|V|^{n}\right)$.

The first of these additional constraints-Property $\left(M 1^{*}\right)$ — puts a polynomial size restriction on the partition of items in the model of group centrality measure for every possible graph. In practice, this means that items cannot have more than a polynomial number of values, as this would prohibit polynomial computation. The second constraint-Property $\left(M 2^{*}\right)$-reduces the overall computational problem to the computation of a number of values. It is necessary to compute the values $\left|R_{\Theta_{l}}(u)\right|,\left|\widetilde{R}_{\Theta_{l}}(u)\right|,\left|N_{\# k}^{-1}\left(\Theta_{l}\right)\right|$, and $\left|R_{\# k}^{-1}\left(\Theta_{l}\right)\right|$ in polynomial time, since this allows us to reduce exponential sums in the definition of semivalues into a polynomial number of multiplications. We show in Section 6 that these restrictions are sufficiently broad to capture all fundamental network centrality measures and their variants known in the literature. Our key result is as follows:

Theorem 1 For all $\psi \in \mathscr{M}^{*}$ and semivalues $\phi, \operatorname{Semivalue}(\psi, \phi)$ is solvable in polynomial time. 
Proof: In order to solve $\operatorname{Semivalue}(\psi, \phi)$ in polynomial time, we will use equation (1) and show how to compute $\mathbb{E}_{C_{k}}\left[\mathrm{MC}\left(C_{k}, u\right)\right]$ in polynomial time for all $k$. To this end, we need to consider the contributions of the node $u$ to $C_{k}$ through the various items that $u$ is in relation with (as defined by $R(u), \widetilde{R}(u)$ and $N(u))$.

We will denote by $\operatorname{MC}(C, u, \vartheta)$ the marginal contribution of node $u$ to $C \backslash\{u\}$ through the item $\vartheta$ (which we will define below). In effect, we have

$$
\operatorname{MC}(C, u)=\sum_{\vartheta \in \Theta_{G}} \operatorname{MC}(C, u, \vartheta) .
$$

Two computational issues arise. First, we cannot immediately use this result, as it would require iteration over all coalitions. Second, iterating over a possibly exponential number of items within this sum is quite troubling. To address the first issue, we will denote by $\operatorname{MC}(k, u, \vartheta)$ the sum of contributions of $u$ through $\vartheta$ to all coalitions of size $k$. To address the second, for all groups $\Theta_{l}$ we will consider instead $\operatorname{MC}\left(k, u, \Theta_{l}\right)=\sum_{\vartheta \in \Theta_{l}} \operatorname{MC}(k, u, \vartheta)$. We get:

$$
\mathbb{E}_{C_{k}}\left[\mathrm{MC}\left(C_{k}, u\right)\right]=\sum_{1 \leq l \leq h(G)} \mathbb{E}_{C_{k}}\left[\mathrm{MC}\left(C_{k}, u, \Theta_{l}\right)\right]=\sum_{1 \leq l \leq h(G)} \frac{\mathrm{MC}\left(k, u, \Theta_{l}\right)}{\left(\begin{array}{c}
|V|-1 \\
k
\end{array}\right)}
$$

The remainder of the proof will focus on defining $\operatorname{MC}(C, u, \vartheta)$ and computing $\operatorname{MC}(k, u, \vartheta)$. Polynomial computation of $\mathrm{MC}\left(k, u, \Theta_{l}\right)$ will follow. A node $u$ can contribute (possibly negative) value to a coalition $C$ through $\vartheta \in \Theta_{l}$ in one of three mutually exclusive ways. These are:

[1] $R(u, \vartheta)$ and $N(C, \vartheta)$,

[2] $\widetilde{R}(u, \vartheta)$ and $R(C, \vartheta)$,

[3] $R(u, \vartheta)$ or $N(u, \vartheta)$, and $R(C, \vartheta)$.

For all other cases $\operatorname{MC}(C, u, \vartheta)=0$. Since the remainder of the proof hinges on the understanding of these cases, let us illustrate them with our running example from Figure 3.

Example 6 Let $S=\{a\}$. We will compute the contribution of node b to coalition $S$. We have $\operatorname{MC}\left(S, b, \vartheta_{1}\right)=0$, since $\widetilde{R}\left(S, \vartheta_{1}\right)$ (meaning there is nothing to take away). Evidently, none of the cases [1], [2], [3] are satisfied. We also have $\widetilde{R}\left(b, \vartheta_{3}\right)$ and $N\left(S, \vartheta_{3}\right)$, which implies $M C\left(S, b, \vartheta_{3}\right)=$ 0 . A contribution can only be made through the item $\vartheta_{2}$. We have: $R\left(b, \vartheta_{2}\right)$ and $R\left(S, \vartheta_{2}\right)$, which satisfies condition [3]. It is not immediately obvious why a contribution is made, since $\vartheta_{2}$ is already positively related with $S$. However, it is necessary to keep in mind that the size of the coalition will change. Due to the function $g$, this means that the value added by item $\vartheta_{2}$ will now be $g(k+1) f\left(\vartheta_{2}\right)$ instead of $g(k) f\left(\vartheta_{2}\right)$. We say, then, that b contributes the value $g(k+1) f\left(\vartheta_{2}\right)-g(k) f\left(\vartheta_{2}\right)$ through the item $\vartheta_{2}$ to coalition $S$.

We compute $\mathrm{MC}(k, u, \vartheta)$ in these three cases. In equations (3), (4) and (5) we will use properties (M2) and (M3) to give a closed formula that depends just on $\Theta_{l}$ and the size of the coalition, but not on $\vartheta$ itself. These formulas can be computed in polynomial time due to $\left(M 2^{*}\right)$.

For the first case, $\operatorname{MC}(C, u, \vartheta)=g(k+1) f(\vartheta)$. There are $\left|N_{\# k}^{-1}(\vartheta)\right|$ coalitions of size $k$ such that $N(C, \vartheta)$. Thus, for $\vartheta \in \Theta_{l}$ we have:

$$
\operatorname{MC}^{[1]}(k, u, \vartheta)=g(k+1) f(\vartheta)\left|N_{\# k}^{-1}(\vartheta)\right|=g(k+1) f\left(\Theta_{l}\right)\left|N_{\# k}^{-1}\left(\Theta_{l}\right)\right| .
$$


For the second case, $\operatorname{MC}(C, u, \vartheta)=-g(k) f(\vartheta)$. There are $\left|R_{\# k}^{-1}(\vartheta)\right|$ coalitions of size $k$ such that $R(C, \vartheta)$. We have:

$$
\mathrm{MC}^{[2]}(k, u, \vartheta)=-g(k) f(\vartheta)\left|R_{\# k}^{-1}(\vartheta)\right|=-g(k) f\left(\Theta_{l}\right)\left|R_{\# k}^{-1}\left(\Theta_{l}\right)\right| .
$$

For the third case, $\mathrm{MC}^{[3]}(C, u, \vartheta)=\Delta^{k} f(\vartheta)$, where $\Delta^{k}=g(k+1)-g(k)$. There are exactly $\left|R_{\# k}^{-1}(\vartheta)\right|$ coalitions of size $k$ such that $R(C, \vartheta)$. We therefore have:

$$
\operatorname{MC}^{[3]}(k, u, \vartheta)=\Delta^{k} f(\vartheta)\left|R_{\# k}^{-1}(\vartheta)\right|=\Delta^{k} f\left(\Theta_{l}\right)\left|R_{\# k}^{-1}\left(\Theta_{l}\right)\right| .
$$

Since $\operatorname{MC}^{[i]}(k, u, \vartheta)$ depends on the group $\Theta_{l}$ such that $\vartheta \in \Theta_{l}$, equivalently we write $\operatorname{MC}^{[i]}\left(k, u, \Theta_{l}\right)$. Using equations (3), (4) and (5), we find that $\operatorname{MC}\left(k, u, \Theta_{l}\right)$ equals

$$
\operatorname{MC}\left(k, u, \Theta_{l}\right)=\sum_{\vartheta \in R_{\Theta_{l}}(u)} \operatorname{MC}^{[1]}(k, u, \vartheta)+\sum_{\vartheta \in \widetilde{R}_{\Theta_{l}}(u)} \operatorname{MC}^{[2]}(k, u, \vartheta)+\sum_{\vartheta \in R_{\Theta_{l}}(u) \cup N_{\Theta_{l}}(u)} \operatorname{MC}^{[3]}(k, u, \vartheta)
$$

Using equations (3), (4) and (5) this term can be rewritten as

$$
\begin{aligned}
\operatorname{MC}\left(k, u, \Theta_{l}\right)= & \left|R_{\Theta_{l}}(u)\right| \operatorname{MC}^{[1]}\left(k, u, \Theta_{l}\right)+\left|\widetilde{R}_{\Theta_{l}}(u)\right| \operatorname{MC}^{[2]}\left(k, u, \Theta_{l}\right) \\
& +\left(\left|R_{\Theta_{l}}(u)\right|+\left|N_{\Theta_{l}}(u)\right|\right) \operatorname{MC}^{[3]}\left(k, u, \Theta_{l}\right) .
\end{aligned}
$$

Observe that the summations over the potentially exponentially growing sets $R_{\Theta_{l}}(u), \widetilde{R}_{\Theta_{l}}(u)$, and $N_{\Theta_{l}}(u)$ fall out of the equation. Moreover, in virtue of property $\left(M 2^{*}\right)$, this term can be computed in polynomial time.

Finally, equations (6), (2) and (1) yield the following closed formula for the semivalue of node $v$, which also furnishes us with a polynomial algorithm and concludes the proof:

$$
\phi_{v}(\nu)=\sum_{0 \leq k<|V|} \beta(k) \sum_{1 \leq l \leq h(G)} \frac{\mathrm{MC}\left(k, u, \Theta_{l}\right)}{\left(\begin{array}{c}
|V|-1 \\
k
\end{array}\right)} .
$$

This concludes the proof.

Of course, since the Shapley value and Banzhaf index are instances of semivalues, we can also obtain the following:

Corollary 1 For all $\psi \in \mathscr{M}^{*}$ we can compute the Shapley value-based and Banzhaf centralities in polynomial time.

Equation 7 in the proof of Theorem 1 yields an algorithm to compute $\operatorname{SemiVALuE}(\psi, \phi)$. Algorithm 1 (SEMI) is a direct implementation. In lines 5-7, 8-10 and 11-12, it computes the contribution of $v$ to $C_{k}$ associated with the expressions $\operatorname{MC}^{[1]}\left(k, v, \Theta_{l}\right), \mathrm{MC}^{[2]}\left(k, v, \Theta_{l}\right)$ and $\mathrm{MC}^{[3]}\left(k, v, \Theta_{l}\right)$, respectively. Recall that polynomial computation of $\left|N_{\# k}^{-1}\left(\Theta_{l}\right)\right|$ and $\left|R_{\# k}^{-1}\left(\Theta_{l}\right)\right|$ was one of our assumptions in $\mathscr{M}^{*}$. The functions for computing these values are represented in the pseudocode by $c_{G}^{N}$ and $c_{G}^{R}$ and must be provided in the input. The values $\left|R_{\Theta_{l}}(\{v\})\right|$ and $\left|\widetilde{R}_{\Theta_{l}}(\{v\})\right|$ must be precomputed for all $1 \leq l \leq h(G)$ and $v \in V$. Given those precomputations, $\left|N_{\Theta_{l}}(\{v\})\right|$ can also be computed in constant time. Given this, we can draw the following additional conclusion as to the asymptotic complexity of Algorithm 1. 


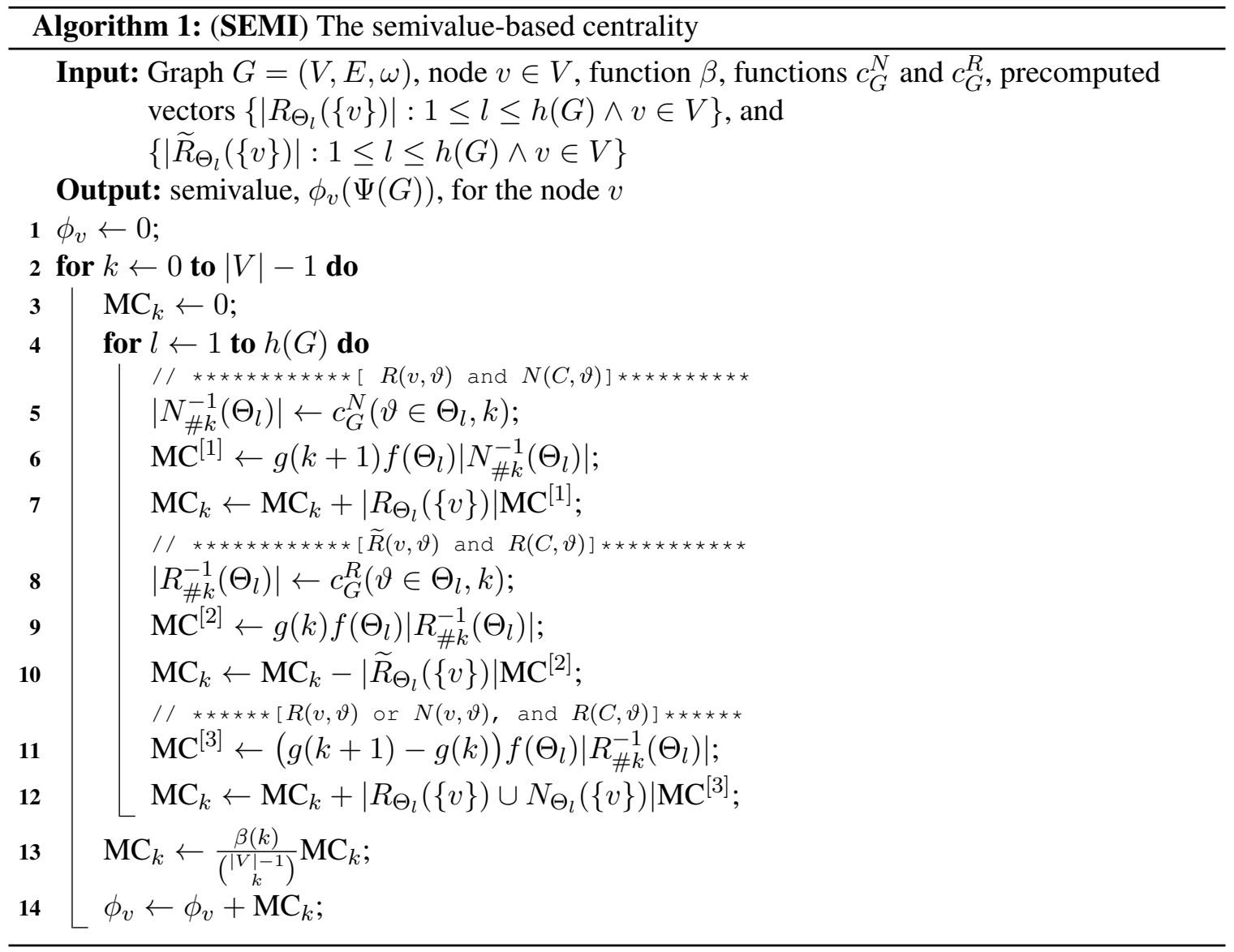

Corollary 2 If $\psi \in \mathscr{M}^{*}$, then there exist constants $m_{1}, m_{2}$, and $m_{3}$ such that

1. $f$ can be computed in time $O\left(|V|^{m_{1}}\right)$;

2. g can be computed in time $O\left(|V|^{m_{2}}\right)$;

3. $\left|N_{\# k}^{-1}\left(\Theta_{l}\right)\right|,\left|R_{\# k}^{-1}\left(\Theta_{l}\right)\right|,\left|R_{\Theta_{l}}(u)\right|$ and $\left|\widetilde{R}_{\Theta_{l}}(u)\right|$ can be computed in time $O\left(|V|^{m_{3}}\right)$.

For a given network $G=(V, E)$ and node $u$, Algorithm 1 runs in time

$$
O\left(h(G)|V|^{m_{1}}+|V|^{m_{2}+1}+h(G)|V|^{m_{3}+1}\right) .
$$

The orders of $m_{1}, m_{2}$, and $m_{3}$ depend on the specific centrality that is being considered. In the next section we show that for parametrised group degree, betweenness and closeness centralities we have $m_{1}=m_{2}=m_{3}=0$. In the case of betweenness and closeness, however, we first need to perform some precomputations that take $O\left(|V|^{2} \log (|V|)+|V||E|\right)$ time. However, since in both cases $h(G)=|V|^{2}$, then $h(G)|V|^{m_{3}+1}=|V|^{3}$, it means that these precomputations do not impact the overall complexity of computing the centralities. 


\section{Computational Analysis of Semivalues for Parametrised Group Degree, Betweenness and Closeness Centralities}

In this section, we show that our framework can be applied to a wide variety of group centrality measures. We identify efficiently computable game-theoretic centrality measures that correspond to the semivalue of parametrised group degree, parametrised group betweenness, and parametrised group closeness. These parametrised measures are more general than those presented earlier and define a classes of group centrality measures based on degree, betweenness and closeness. Such an approach allows us to generalise the computational results in the literature to whole classes rather than individual measures. The other obvious generalisation of our work in comparison to the literature is that previous work has usually focused on just a single semivalue such as the Shapley value or the Banzhaf index. For example, Szczepański et al. $(2012,2016)$ developed polynomialtime algorithms to compute the Shapley value of betweenness centrality, and Michalak et al. (2013b) did so for both group degree and generalised closeness centrality. The remainder of this section is devoted to analysing the parametrised measures. We show how to build the model for each group centrality in our framework and then derive the individual components and functions required to invoke the method SEMI in Algorithm 1 in order to compute the semivalue of each measure.

\subsection{Parametrised Degree Centrality}

In this section, we define in our framework the class of cooperative games, where a node's value is based on its degree. We consider the general parametrised group degree centrality of a coalition $C$ in graph $G$, which is defined by:

$$
\psi^{D}(G)(C)=\nu_{G}^{D}(C)=g(|C|) \sum_{v \in E(C)} f(v)
$$

where $E(C)$ is the set of neighbours of $C$, and $f$ and $g$ are polynomially computable parameters such that $\operatorname{deg}(u)=\operatorname{deg}(v)$ implies $f(u)=f(v)$ for all $u, v \in V$. Using this characteristic function, we build semivalue degree, semivalue weighted degree, semivalue impact factor and semivalue normalised degree centralities (Table 3).

Proposition 1 The parametrised group centrality measure $\psi^{D}$ (for polynomially computable parameters $f$ and $g$ ) belongs to $\mathscr{M}^{*}$. Consequently, $\operatorname{Semivalue}\left(\psi^{D}, \phi\right)$ can be solved in polynomial time and the corresponding semivalue-based centrality can be computed in time $O\left(|V|^{2}+|V|^{m_{1}+1}+\right.$ $\left.|V|^{m_{2}+1}\right)$.

Proof: We will prove that for every $\nu_{G}^{D}$ we can find an $M_{G}=\left(\left\{\Theta_{l}\right\}_{l=1}^{h(G)}, R, \widetilde{R}, N, f, g\right)$ that models it, proving $\psi^{D}(G)$ is in the class $\mathscr{M}$. Next, we will show that all properties necessary for $\mathscr{M}^{*}$ are also satisfied. To this end, for all graphs $G$ we define the set of items $\Theta_{G}=V$. The two relations, $R, \widetilde{R} \subseteq V \times V$, will be as follows:

$$
\begin{aligned}
& R(v, u) \text { if and only if } \exists_{(v, u) \in E} v \neq u \\
& \widetilde{R}(v, u) \text { if and only if } v=u .
\end{aligned}
$$

We define the partitions of $\Theta_{G}$ by $\Theta_{l}=\{v \in V: \operatorname{deg}(v)=l\}$. Hence, we can rewrite $\nu_{G}^{D}(C)=$ $g(|C|) \sum_{\vartheta \in R(C)} f(\vartheta)$, satisfying property $(M 1)$. (M2) is satisfied due to our assumption on $f$ and 


\begin{tabular}{llccc}
\hline Centrality measure & Introduced in & $f(v)$ & $g(|C|)$ & Complexity \\
\hline degree $^{(*)}$ & Everett and Borgatti (1999) & 1 & 1 & $O\left(|V|^{2}\right)$ \\
weighted degree & Newman (2004) & $\frac{1}{\operatorname{deg}(v)}$ & 1 & $O\left(|V|^{2}\right)$ \\
impact factor & Bollen, Sompel, Smith, and Luce (2005) & 1 & $\frac{1}{|C|}$ & $O\left(|V|^{2}\right)$ \\
normalised degree & Everett and Borgatti (1999) & 1 & $\frac{1}{|V \backslash C|}$ & $O\left(|V|^{2}\right)$ \\
\hline
\end{tabular}

${ }^{(*)}$ Michalak et al. (2013b) presented the first algorithm for Shapley value degree centrality, whereas our algorithm applies to all semivalues.

Table 3: Results for group degree centrality. The functions $f$ and $g$ for various group degree centralities and where they were first introduced. The semivalues of these group centralities can be computed for the first time in polynomial time using our framework.

all the examples in Table 3 satisfy it as well. Next, we consider the relations with respect to coalition sizes $k$ and for $v \in \Theta_{G}$ we have:

$$
\begin{aligned}
& \left|N_{\# k}^{-1}(v)\right|= \begin{cases}0 & \text { if }|V|-1-\operatorname{deg}(v)<k \\
\left(\begin{array}{l}
|V|-1-\operatorname{deg}(v) \\
k
\end{array}\right) & \text { otherwise. }\end{cases} \\
& \left|R_{\# k}^{-1}(v)\right|= \begin{cases}0 & \text { if }|V|=k \\
\left(\begin{array}{c}
|V| \\
k
\end{array}\right)-\left|N_{\# k}^{-1}(v)\right|-\left(\begin{array}{c}
|V|-1 \\
k-1
\end{array}\right) & \text { otherwise. }\end{cases}
\end{aligned}
$$

Note that for all $\Theta_{l}$ and all $\vartheta_{i}, \vartheta_{j} \in \Theta_{l}$ we have that $\left|N_{\# k}^{-1}\left(\vartheta_{i}\right)\right|=\left|N_{\# k}^{-1}\left(\vartheta_{j}\right)\right|$ and $\left|R_{\# k}^{-1}\left(\vartheta_{i}\right)\right|=$ $\left|R_{\# k}^{-1}\left(\vartheta_{j}\right)\right|$, satisfying $(M 3)$ and $(M 4)$. Hence, $\psi \in \mathscr{M}$.

Regarding $\mathscr{M}^{*}$, property $\left(M 1^{*}\right)$ holds trivially, since clearly $h(G) \leq|V|$. Both $f$ and $g$ can be computed in $O(1)$ time once the degree of each node is precomputed, so we have $m_{1}=m_{2}=0$. For each $v \in V$ and $1 \leq l \leq h(G)$ it is easy to compute $\left|R_{\Theta_{l}}(\{v\})\right|$ and $\mid \widetilde{R}_{\Theta_{l}}(\{v\} \mid$. To do so, we must first compute the degree of each node, which takes $O(|V|+|E|)$ time. Next, the number of neighbours of $v$ with degree $l$ must be counted to compute $\left|R_{\Theta_{l}}(\{v\})\right|$, and $\left|\widetilde{R}_{\Theta_{l}}(\{v\})\right|=1$ if and only if $l=\operatorname{deg}(v)$ and $\left|\widetilde{R}_{\Theta_{l}}(\{v\})\right|=0$ otherwise. These computations can be done in $O(1)$ time. Finally, $\left|N_{\# k}^{-1}(\vartheta)\right|$ and $\left|R_{\# k}^{-1}(\vartheta)\right|$ can be computed in $O(1)$ time simply from their definitions shown above, satisfying property $\left(M 2^{*}\right)$. With this, for $m_{3}$ from Corollary 2 we have $m_{3}=0$.

Due to Theorem 1, we can compute semivalue-based centralities based on $v_{G}^{D}$ in polynomial time. Due to Corollary 2, we can compute $\phi_{v}\left(\nu_{G}^{D}\right)$ in time $O\left(|V|^{2}+|V|^{m_{1}+1}+|V|^{m_{2}+1}\right)$.

Algorithm 3 is presented in Appendix B.1 and invokes the method SEMI (Algorithm 1) in order to compute parametrised degree centrality. Our algorithm takes as an argument the graph $G$, defines the two functions, $c_{G}^{N}$ and $c_{G}^{R}$. They compute $\left|N_{\# k}^{-1}\left(\Theta_{l}\right)\right|$ and $\left|R_{\# k}^{-1}\left(\Theta_{l}\right)\right|$, respectively, in polynomial time. Note that Algorithm 3 is also parametrised by the probability distribution $\beta$, which allows for the computation of any semivalue-based centrality. Pre-computing $\left|R_{\Theta_{l}}(\{v\})\right|$ for each node $v$ comes down to computing the number of neighbours of $v$ with degree $l$. This operation takes no more than $O\left(|V|^{2}\right)$ time. Precomputing $\left|\widetilde{R}_{\Theta_{l}}(\{v\})\right|$ is trivial, since it is always either equal to 0 or 1 . Thus, these pre-computations take $O\left(|V|^{2}\right)$ time. Due to Corollary 2, this algorithm runs in $O\left(|V|^{2}+|V|^{m_{1}+1}+|V|^{m_{2}+1}\right)$ time (recall that $m_{1}$ and $m_{2}$ are such that $f$ can be computed in $O\left(|V|^{m_{1}}\right)$ time and $g$ can be computed in $O\left(|V|^{m_{2}}\right)$ time). 


\begin{tabular}{llccc}
\hline Centrality measure & Introduced in & $f(s, t)$ & $g(|C|)$ & Complexity \\
\hline stress $^{(*)}$ & Szczepański et al. (2012) & 1 & 1 & $O\left(|V|^{3}\right)$ \\
betweenness $^{(*)}$ & Everett and Borgatti (1999) & $\frac{1}{\sigma_{s t}}$ & 1 & $O\left(|V|^{3}\right)$ \\
distance-scaled betw. & Brandes (2008) & $\frac{1}{\sigma_{s t} \operatorname{dist}(s, t)}$ & 1 & $O\left(|V|^{3}\right)$ \\
normalised betw. & Everett and Borgatti (1999) & $\frac{1}{\sigma_{s t}}$ & $\frac{2}{|V \backslash C|(|V \backslash C|-1)}$ & $O\left(|V|^{3}\right)$ \\
\hline
\end{tabular}

${ }^{*}$ Szczepański et al. $(2012,2016)$ presented the first algorithm for Shapley value stress and betweenness centralities, whereas our algorithm applies to all semivalues.

Table 4: Results for group betweenness centrality. The functions $f$ and $g$ for various group betweenness centralities and where they were first introduced. The semivalues of these group centralities can be computed for the first time in polynomial time using our framework.

\subsection{Parametrised Betweenness Centrality}

In this subsection, we show how to express in our framework the class of cooperative games, where a node's value is based on its betweenness centrality. Recall that we denote the set of paths between $s$ and $t$ by Paths $(s, t)$ and the set of all shortest paths in $G$ by $\operatorname{SP}(G)$. We denote by $\sigma_{s t}$ the number of shortest paths between $s$ and $t$ (if $s=t$ then $\sigma_{s t}=1$ ) and by $\sigma_{s t}(C)$ the number of shortest paths between $s$ and $t$ that pass through some node $v \in C$.

The general parametrised group betweenness centrality of a coalition $C$ in graph $G$ is defined by:

$$
\psi^{B}(G)(C)=\nu_{G}^{B}(C)=g(|C|) \sum_{s, t \notin C} \sigma_{s t}(C) f(s, t),
$$

where $f$ and $g$ polynomially computable parameters. ${ }^{8}$ Using this characteristic function, we build semivalue stress, semivalue betweenness, semivalue distance-scaled betweenness and semivalue normalised betweenness centralities (Table 4).

Proposition 2 The parametrised group centrality measure $\psi^{B}$ (for polynomially computable parameters $f$ and $g$ ) belongs to $\mathscr{M}^{*}$. Consequently, $\operatorname{SemiVALUE}\left(\psi^{B}, \phi\right)$ can be solved in polynomial time and the corresponding semivalue-based centrality can be computed in time $O\left(|V|^{3}+|V|^{m_{1}+2}+\right.$ $\left.|V|^{m_{2}+1}\right)$.

Proof: First we will prove that for every $\nu_{G}^{B}$ we can find an $M_{G}=\left(\left\{\Theta_{l}\right\}_{l=1}^{h(G)}, R, \widetilde{R}, f, g\right)$ that models it and satisfies the conditions $(M 1)$ through $(M 4)$ for class $\mathscr{M}$. Next, we will show that all properties of class $\mathscr{M}^{*}$ are also satisfied. To this end, we define $\Theta_{G}=S P(G)$ (set of all shortest paths in graph $G$ ). Also, we define the two relations, $R, \widetilde{R} \subseteq V \times S P(G)$, as follows:

$$
\begin{aligned}
& R\left(v, \pi_{s t}\right) \quad \text { if and only if } \quad v \in \pi_{s t} \text { and } v \neq s \text { and } v \neq t \\
& \widetilde{R}\left(v, \pi_{s t}\right) \text { if and only if } \quad v=s \text { or } v=t .
\end{aligned}
$$

and we define the set of partitions of $\Theta_{G}$ as:

$$
\Theta_{s t}=\left\{\pi_{s t} \in S P(G): \pi_{s t} \in \operatorname{Paths}(s, t)\right\} .
$$

8. If $\sigma_{x y}\left(C^{\prime}\right)=0$, we postulate $\frac{0}{0}=0$. 
We slightly abuse notation here by indexing groups of items by $s, t$ for $s, t \in V$. We refer to each group of items therefore by $\Theta_{s, t}$ and define it as the set of all the shortest paths between $s$ and $t$. In this way $\nu_{G}^{B}(C)=g(|C|) \sum_{\vartheta \in R(C)} f(\vartheta)$, satisfying properties $(M 1)$ and $(M 2)$. Next, we look at the three relations with respect to coalitions of different sizes. The set of nodes $C$ is in relation $N$ with shortest path $\pi_{s t} \in S P(G)$ if and only if $\pi_{s t} \cap C=\emptyset$, so we get:

$$
\left|N_{\# k}^{-1}\left(\pi_{s t}\right)\right|= \begin{cases}0 & \text { if }|V|-\left|\pi_{s t}\right|<k \\
\left(\begin{array}{c}
|V|-\left|\pi_{s t}\right| \\
k
\end{array}\right) & \text { otherwise. }\end{cases}
$$

Let $e\left(\pi_{s t}\right)=\min \left(2,\left|\pi_{s t}\right|\right)$. Then,

$$
\left|\widetilde{R}_{\# k}^{-1}\left(\pi_{s t}\right)\right|= \begin{cases}0 & \text { if } k=0 \\
e\left(\pi_{s t}\right) & \text { if } k=1 \\
1 & \text { if } k=|V| \\
\sum_{i=1}^{e\left(\pi_{s t}\right)}\left(\begin{array}{c}
e\left(\pi_{s t}\right) \\
i
\end{array}\right)\left(\begin{array}{c}
|V|-e\left(\pi_{s t}\right) \\
k-i
\end{array}\right) & \text { otherwise. }\end{cases}
$$

Since all coalitions are exclusively in relation $R, \widetilde{R}$ or $N$ with every item, we have:

$$
\left|R_{\# k}^{-1}\left(\pi_{s t}\right)\right|=\left(\begin{array}{c}
|V| \\
k
\end{array}\right)-\left|N_{\# k}^{-1}\left(\pi_{s t}\right)\right|-\left|\widetilde{R}_{\# k}^{-1}\left(\pi_{s t}\right)\right| .
$$

Properties (M3) and (M4) may seem troubling at first, but note that all shortest paths between two nodes $s$ and $t$ must necessarily be of the same length. Therefore, $\psi^{B} \in \mathscr{M}$.

We note that $h(G)=|V|^{2}$, satisfying property $\left(M 1^{*}\right)$. Property $\left(M 2^{*}\right)$ is satisfied by our assumption on $f$ and $g$ and for all functions in Table 4. Moreover, for each $v \in V$ and $s, t \in V$ it is easy to precompute $\left|R_{\Theta_{s, t}}(\{v\})\right|$ and $\left|\widetilde{R}_{\Theta_{s, t}}(\{v\})\right|$ for some $v \in V$ and all $s, t \in V$ in time $O\left(|V|^{2}+\right.$ $|V||E|)$ using the BFS algorithm (Cormen, Stein, Rivest, \& Leiserson, 2001) for unweighted graphs, and in time $O\left(|V|^{2} \log (|V|)+|V||E|\right)$ using the Dijkstra algorithm (Dijkstra, 1959) for weighted graphs, again satisfying $\left(M 2^{*}\right)$. An example of how to perform such computations for all nodes in a graph is presented in Appendix B.2 in Algorithm 4. Once these computations are done, $\left|R_{\Theta_{s, t}}(\{v\})\right|$ and $\left|\widetilde{R}_{\Theta_{s, t}}(\{v\})\right|$ can be computed in $O(1)$ time. For each $\pi_{s t} \in \Theta_{G}$ and $k \in\{0, \ldots,|V|-1\}$ we can compute $\left|N_{\# k}^{-1}\left(\pi_{s t}\right)\right|$ and $\left|R_{\# k}^{-1}\left(\pi_{s t}\right)\right|$ in $O(1)$ time simply from the characterisations of these sets that we have shown above (a modified BFS or Dijkstra algorithm can return the value of $\left.e\left(\pi_{s t}\right)=\min \left(2,\left|\pi_{s t}\right|\right)\right)$, which satisfies $\left(M 2^{*}\right)$. This also means that for $m_{3}$ from Corollary 2 we have $m_{3}=0$.

It follows that $\psi^{B} \in \mathscr{M}^{*}$ and due to Theorem 1 we can compute any semivalue-based centrality based on the game $v_{G}^{B}$ in polynomial time. Due to Corollary 2, we can compute $\phi_{v}\left(\nu_{G}^{B}\right)$ for the node $v \in V$ in time $O\left(|V|^{3}+|V|^{m_{1}+2}+|V|^{m_{2}+1}\right)$.

Algorithm 5 is presented in Appendix B.2 and computes the semivalue betweeness centrality.

\subsection{Parametrised Closeness Centrality}

The last class of characteristic functions that we will define in our framework assigns a value to a node based on its distance to other nodes. The parametrised closeness centrality is defined as follows:

$$
\psi^{C L}(G)(C)=\nu_{G}^{C L}(C)=g(|C|) \sum_{v \in V} f(\operatorname{dist}(C, v))
$$




\begin{tabular}{llccc}
\hline Centrality measure & Introduced in & $f(d)$ & $g(|C|)$ & Complexity \\
\hline closeness & Everett and Borgatti (1999) & 1 & 1 & $O\left(|V|^{3}\right)$ \\
harmonic $^{(*)}$ & Boldi and Vigna (2014) & $\frac{1}{d}$ & 1 & $O\left(|V|^{3}\right)$ \\
influence game $^{(*)}$ & Michalak et al. (2013b) & $\begin{array}{c}\text { positive value } \\
\text { decreasing }\end{array}$ & 1 & $O\left(|V|^{3}\right)$ \\
normalised closeness & Everett and Borgatti (1999) & $\frac{1}{d}$ & $|V \backslash C|$ & $O\left(|V|^{3}\right)$ \\
\hline
\end{tabular}

${ }^{(*)}$ Michalak et al. (2013b) presented the first algorithm for Shapley value harmonic and influence game centralities, whereas our algorithm applies to all semivalues.

Table 5: Results for group closeness centrality. The functions $f$ and $g$ for various group closeness centralities and where they were first introduced. The semivalues of these group centralities can be computed for the first time in polynomial time using our framework.

where $f$ and $g$ are polynomially computable parameters. Using this characteristic function, we build semivalue closeness, semivalue harmonic, semivalue influence game and semivalue normalised closeness centralities (Table 5).

Proposition 3 The parametrised group centrality measure $\psi^{C L}$ (for polynomially computable parameters $f$ and $g)$ belongs to $\mathscr{M}^{*}$. Consequently, $\operatorname{SemivaLuE}\left(\psi^{C L}, \phi\right)$ can be solved in polynomial time and the corresponding semivalue-based centrality can be computed in time $O\left(|V|^{3}+|V|^{m_{1}+2}+\right.$ $\left.|V|^{m_{2}+1}\right)$.

Proof: First we prove that for every $\nu_{G}^{C L}$ we can find an $M_{G}=\left(\left\{\Theta_{l}\right\}_{l=1}^{h(G)}, R, \widetilde{R}, f, g\right)$ that models it, satisfying the necessary conditions for class $\mathscr{M}$. Next, we will prove that all properties of class $\mathscr{M}^{*}$ are also satisfied. To this end, we will define items as pairs of the form $\langle u, d\rangle \subseteq V \times \operatorname{dists}(G)$, where dists $(G)$ is the set of distances between any pair of nodes in $G$. They will represent nodes and all possible distances between them. We define $\Theta_{G}=\left\{\langle u, d\rangle \in V \times \mathbb{N}: \exists_{v \in V} \operatorname{dist}(u, v)=d\right\}$ and the partition $\left\{\{\vartheta\} \mid \vartheta \in \Theta_{G}\right\}$ of singletons. We slightly abuse notation here by indexing groups of items (i.e., sets in the partition) by $u, d$. We refer to a group of items therefore by $\Theta_{u, d}$. Next, we define the two relations, $R, \widetilde{R} \subseteq V \times V \times\{0, \ldots,|V|-1\}$, as follows:

$$
\begin{aligned}
& R(v,\langle u, d\rangle) \text { if and only if } \operatorname{dist}(v, u)=d \\
& \widetilde{R}(v,\langle u, d\rangle) \text { if and only if } \operatorname{dist}(v, u)<d .
\end{aligned}
$$

In this way $\nu_{G}^{C L}(C)=g(|C|) \sum_{\vartheta \in R(C)} f(\vartheta)$, satisfying $(M 1) .{ }^{9}$ Also $(M 2)$ holds for all examples in Table 5, and (M3) and (M4) hold trivially since groups of items are singletons. Thus, $\psi^{C L} \in \mathscr{M}$.

Property $\left(M 1^{*}\right)$ holds, since $h(G)$ is bounded by $|V|^{2}$ (number of nodes and all possible distances between them). Functions $f$ and $g$ can be computed in $O(1)$ time and, therefore, $\left(M 2^{*}\right)$ also holds. This also means that $m_{1}=m_{2}=0$. We note that $h(G) \lesseqgtr|V|^{2}$ and that for each $v \in V$ and $1 \leq d \leq h(G)$ it is easy to precompute $\left|R_{\Theta_{u, d}}(\{v\})\right|$ and $\left|\widetilde{R}_{\Theta_{u, d}}(\{v\})\right|$ with the help of the BFS algorithm for unweighted graphs, or the Dijkstra algorithm for weighted graphs, thus satisfying $\left(M 2^{*}\right)$. Once the distances between all nodes are computed either using BFS or the Dijkstra

9. In our examples $f$ ignores the first parameter of $\vartheta$ and is simply a function of distance. 
algorithm, $\left|R_{\Theta_{u, d}}(\{v\})\right|$ is equal to 1 if $\operatorname{dist}(v, u)=d$ and 0 otherwise, whereas $\left|\widetilde{R}_{\Theta_{u, d}}(\{v\})\right|$ is computed identically with the condition $\operatorname{dist}(v, u)<d$. In both cases, computing these values take $O(1)$ time.

Next, we will consider the three relations with respect to coalition sizes. To this end, we will define

$$
\operatorname{Nod}_{\sim d}(u)=|\{v: \operatorname{dist}(u, v) \sim d\}| \quad \operatorname{NodSet}_{\sim d}(u)=\{v: \operatorname{dist}(u, v) \sim d\} .
$$

where $\sim$ can be either $<$ or $=$. These values are simple to compute for all $u, d$ in $O\left(|V|^{2}+|V||E|\right)$ time using the BFS algorithm for unweighted graphs or $O\left(|V|^{2} \log (|V|)+|V||E|\right)$ time for weighted graphs using the Dijkstra algorithm. Note that:

$$
\begin{aligned}
& R(C,\langle u, d\rangle) \text { iff } \operatorname{NodSet}_{<d}(u) \cap C=\emptyset \text { and } \operatorname{NodSet}_{=d}(u) \cap C \neq \emptyset \\
& \widetilde{R}(C,\langle u, d\rangle) \text { iff } \operatorname{NodSet}_{<d}(u) \cap C \neq \emptyset .
\end{aligned}
$$

Using the above relations we can now calculate:

$$
\begin{aligned}
& \left|R_{\# k}^{-1}(\langle u, d\rangle)\right|=\sum_{i=1}^{N o d_{=d}(u)}\left(\begin{array}{c}
N o d_{=d}(u) \\
i
\end{array}\right)\left(\begin{array}{c}
|V|-N o d_{=d}(u)-N o d_{<d}(u) \\
k-i
\end{array}\right) \\
& \left|\widetilde{R}_{\# k}^{-1}(\langle u, d\rangle)\right|=\sum_{i=1}^{N o d_{<d}(u)}\left(\begin{array}{c}
N o d_{<d}(u) \\
i
\end{array}\right)\left(\begin{array}{c}
|V|-N_{<d}(u) \\
k-i
\end{array}\right) .
\end{aligned}
$$

Since all coalitions are exclusively in relation $R, \widetilde{R}$ or $N$ with every item, we have:

$$
\left|N_{\# k}^{-1}(\langle u, d\rangle)\right|=\left(\begin{array}{c}
|V| \\
k
\end{array}\right)-\left|R_{\# k}^{-1}(\langle u, d\rangle)\right|-\left|\widetilde{R}_{\# k}^{-1}(\langle u, d\rangle)\right| \text {. }
$$

Due to the above characterisations, for each $\vartheta \in \Theta_{G}$ and $k \in\{0, . .,|V|-1\}$, we can compute $\left|N_{\# k}^{-1}(\vartheta)\right|$ and $\mid R_{\# k}^{-1}\left(\vartheta \mid\right.$ in polynomial time $O(|V|)$, thus satisfying $\left(M 2^{*}\right)$. We show in Appendix C.4 how we can improve this by computing all values at once using dynamic programming, to achieve a computation time of $O(1)$, meaning for $m_{3}$ from Corollary 2 we have $m_{3}=0$.

Hence, $\psi^{C L} \in \mathscr{M}^{*}$. Consequently, $\operatorname{Semivalue}\left(\psi^{C L}, \phi\right)$ can be solved in polynomial time. By virtue of Corollary 2, we can compute every semivalue-based centrality for node $v \in V$ in time $O\left(|V|^{3}+|V|^{m_{1}+2}+|V|^{m_{2}+1}\right)$

Algorithm 6 is presented in Appendix B.3 and uses the method SEMI to compute the parametrised closeness centrality of a node. Evaluating the functions $c_{G}^{N}$ and $c_{G}^{R}$ takes $O(1)$ time. Precomputing $\left|N_{u, d}(\{v\})\right|$ and $\left|R_{u, d}(\{v\})\right|$ for each node $v$ can be done with the help of the BFS algorithm for unweighted networks to compute distances between all nodes in the graph, or the Dijkstra algorithm for weighted networks. This takes $O\left(|V|^{2}+|V||E|\right)$ or $\left.O\left(|V|^{2} \log (|V|)+|V||E|\right)\right)$ time, respectively. In particular, for all nodes $v$ and possible distances $l$, we need to store the number of nodes that are closer to $v$ than $l$ and the number of nodes that are at a distance equal to $l$. Due to Corollary 2, this algorithm computes the parametrised closeness centrality for the node $v$ in $O\left(|V|^{3}+|V|^{m_{1}+2}+|V|^{m_{2}+1}\right)$ time.

In the following section, we present algorithms for computing the Banzhaf index (the most prominent semivalue next to the Shapley value) of the parametrised group centrality measures presented in this section, with the additional constraint that $g(|C|)=1$. Focusing on these specific cases allows us to achieve even faster polynomial computation, often by a factor of $O(|V|)$. 


\section{Computational Analysis of the Banzhaf Index for Degree, Closeness, and Betweenness Centrality}

In this section, we show how to compute the Banzhaf index of power of the parametrised measures from Section 6. Although the Algorithms presented in Section 6 can also perform these computations, if we focus on just the Banzhaf index and restrict the model of the characteristic function such that $g(|C|)=1$ (in practice, the proofs work for any constant function), then quantification over $k$ (the size of the coalition) can be removed from the algorithm and computation generally improves by a factor of $O(|V|)$. Let us define

$$
\mathcal{N}^{l}=\sum_{0 \leq k<|V|} \beta(k) \frac{\left|N_{\# k}^{-1}\left(\Theta_{l}\right)\right|}{\left(\begin{array}{c}
|V|-1 \\
k
\end{array}\right)} \quad \mathcal{R}^{l}=\sum_{0 \leq k<|V|} \beta(k) \frac{\left|R_{\# k}^{-1}\left(\Theta_{l}\right)\right|}{\left(\begin{array}{c}
|V|-1 \\
k
\end{array}\right)}
$$

We can then reorder Equation 7 from Theorem 1-see Appendix C.1-and obtain the following equation:

$$
\phi_{v}(\nu)=\sum_{1 \leq l \leq h(G)}\left|R_{\Theta_{l}}(\{u\})\right| f\left(\Theta_{l}\right) \mathcal{N}^{l}-\sum_{1 \leq l \leq h(G)}\left|\widetilde{R}_{\Theta_{l}}(\{u\})\right| f\left(\Theta_{l}\right) \mathcal{R}^{l} .
$$

It should be clear from this that, if computing $\mathcal{N}^{l}$ and $\mathcal{R}^{l}$ can be achieved in constant time for a given group centrality measure, then this will rid us of the quantification over $k$. This leads to a simplification of the general semivalue algorithm (Algorithm 1), which we present in Algorithm 2. This algorithm computes the Banzhaf centrality of a given node, $v$. As in our previous algorithm, $R_{\Theta_{l}}(\{v\})$ and $\widetilde{R}_{\Theta_{l}}(\{v\})$ must be precomputed for every item group index $l$. The specifics of this for every centrality can be found in the previous section. In addition, $\mathcal{N}^{l}$ and $\mathcal{R}^{l}$ must be computed. The complexity of this algorithm is $O\left(h(G)\left(|V|^{m_{1}}+|V|^{m_{4}}\right)\right)$, where $|V|^{m_{1}}$ is the time required to compute $f\left(\Theta_{l}\right)$ and $|V|^{m_{4}}$ is the time required to compute $\mathcal{N}^{l}, \mathcal{R}^{l}, R_{\Theta_{l}}(\{v\})$ and $\widetilde{R}_{\Theta_{l}}(\{v\})$. We discuss the specifics of computing $\mathcal{N}^{l}$ and $\mathcal{R}^{l}$ for each of the parametrised group degree, betweenness and closeness centralities in what follows.

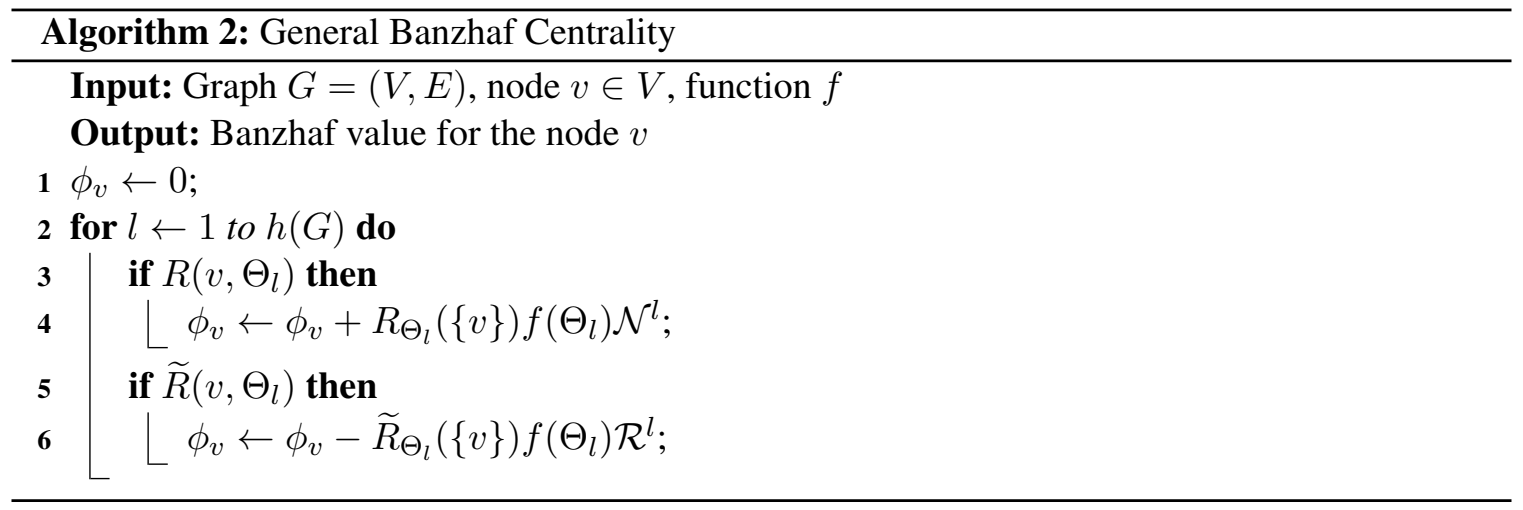




\subsection{A Faster Algorithm for Banzhaf Degree Centrality}

In this section we will show how to compute $\mathcal{N}^{l}$ and $\mathcal{R}^{l}$ for group degree centrality and develop algorithms to compute the Banzhaf index. If $g(|C|)=1$, then this centrality takes the following form:

$$
\psi^{D}(G)(C)=\nu_{G}^{D}(C)=\sum_{v \in E(C)} f(v)
$$

Proposition 4 Given a constant function $g$, $\operatorname{BANZHAF}\left(\psi^{D}, \phi\right)$ can be solved in $O\left(|V|^{1+m_{1}}\right)$ time if $\operatorname{deg}(v)$ can be solved in constant time, and $O\left(|V|^{1+m_{1}}+|E|\right)$ otherwise.

The proof of Proposition 4 can be found in Appendix C.2 and its main finding is as follows:

$$
\mathcal{N}^{l}=\mathcal{N}^{\operatorname{deg}(v)}=\frac{1}{2^{\operatorname{deg}(v)}} \quad \mathcal{R}^{l}=\mathcal{R}^{\operatorname{deg}(v)}=1-\frac{1}{2^{\operatorname{deg}(v)}}
$$

In order to compute the Banzhaf degree centrality these arguments must be passed to Algorithm 2.

\subsection{A Faster Algorithm for Banzhaf Betweenness Centrality}

We will now show how to compute $\mathcal{N}^{l}$ and $\mathcal{R}^{l}$ for the Banzhaf betweenness centrality. First, recall that if $g(|C|)=1$ :

$$
\psi^{B}(G)(C)=\nu_{G}^{B}(C)=\sum_{s, t \notin C} \sigma_{s t}(C) f(s, t)
$$

Proposition 5 Given a constant function $g, \operatorname{BANZHAF}\left(\psi^{B}, \phi\right)$ can be solved in $O\left(|V|^{2+m_{1}}+\right.$ $|V||E|)$ time for undirected graphs, and $O\left(|V|^{2+m_{1}}+|V||E|+|V|^{2} \log (|V|)\right)$ time for directed graphs.

The proof of Proposition 5 can be found in Appendix C.3 and its main finding is as follows:

$$
\mathcal{N}^{s, t}=\frac{2^{|V|-\left|\pi_{s t}\right|}}{2^{|V|-1}}
$$

Assuming $e\left(\pi_{s t}\right)=2$,

$$
\widetilde{\mathcal{R}}^{s t}=\frac{1}{2^{|V|-1}}\left(3\left(2^{|V|-2}\right)-|V|\right)
$$

and assuming $e\left(\pi_{s t}\right)=1$,

$$
\widetilde{\mathcal{R}}^{s, t}=\frac{1}{2},
$$

which leads to:

$$
\mathcal{R}^{s, t}=\frac{2^{|V|}-1}{2^{|V|-1}}-\widetilde{\mathcal{R}}^{s, t}-\mathcal{N}^{s, t}
$$

In order to compute the Banzhaf betweenness centrality these arguments must be passed to Algorithm 2. 


\subsection{A Faster Algorithm for Banzhaf Closeness Centrality}

We will now show how to compute $\mathcal{N}^{l}$ and $\mathcal{R}^{l}$ for the Banzhaf closeness centrality. First, recall that if $g(|C|)=1$ :

$$
\nu_{G}^{C L}(C)=\sum_{v \in V} f(\operatorname{dist}(C, v))
$$

Proposition 6 Given a constant function $g$, BANZHAF $\left(\psi^{C L}, \phi\right)$ can be solved in $O\left(|V|^{2+m_{1}}+\right.$ $|V||E|)$ time for undirected graphs, and $O\left(|V|^{2+m_{1}}+|V||E|+|V|^{2} \log (|V|)\right)$ time for directed graphs.

The proof of Proposition 6 can be found in Appendix C.4 and its main finding is as follows: Assuming $\operatorname{Nod}_{<d}(u)>0$,

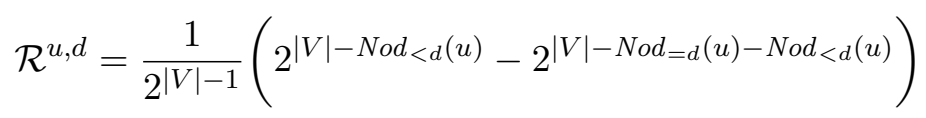

and assuming $\operatorname{Nod}_{<d}(u)=0$,

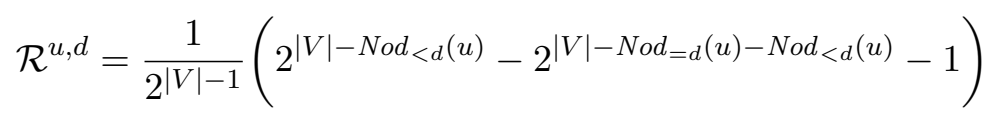

Continuing, if $\operatorname{Nod}_{<d}(u)>0$

$$
\widetilde{\mathcal{R}}^{u, d}=\frac{1}{2^{|V|-1}}\left(2^{|V|}-1-2^{|V|-N_{o d}(u)}\right) .
$$

and if $\operatorname{Nod}_{<d}(u)=0$,

$$
\widetilde{\mathcal{R}}^{u, d}=\frac{1}{2^{|V|-1}}\left(2^{|V|}-2^{|V|-\operatorname{Nod}_{<d}(u)}\right),
$$

which allows us to compute

$$
\mathcal{N}^{u, d}=\frac{2^{|V|}-1}{2^{|V|-1}}-\mathcal{R}^{u, d}-\widetilde{\mathcal{R}}^{u, d}
$$

In order to compute the Banzhaf closeness centrality these arguments must be passed to Algorithm 2.

\section{Conclusions and Future Work}

In this paper, we have proposed a general framework to facilitate computation of semivalues for a wide range of game-theoretic network centrality measures. Whereas previous work has focused on computing some particular solution concept for a given characteristic function, we have shown that it is possible to consolidate and generalise certain computational properties. Given that the model satisfies just a few properties, we show that our algorithm runs in polynomial time. In fact, our algorithm covers all previously proposed centrality measures based on some semivalue (see Table 1) and many more (see Tables 3, 4 and 5). We trust that this will facilitate the study of computational properties and implementation of such measures in the future. 
A number of future research directions stem from this work. First, we would like to investigate whether it would be possible to broaden the scope of our framework. For example, it may be possible to expand the class $\mathscr{M}^{*}$ to include additional group centrality measures. Another direction is to study the computation complexity of computing single-valued solution concepts other than semivalues and the classes of group centrality measures for which these solution concepts can be computed in polynomial time. There are many solution concepts in the literature on game theory, such as probabilistic values, quasivalues, weighted values, indices of power (Monderer \& Samet, 2002), the core (Gillies, 1953, 1959) and nucleolus (Schmeidler, 1969) some of which have not yet been applied to networks or centrality.

Second, it may be possible to use game-theoretic approaches in order to analyse more general types of networks. For example, it may be possible to incorporate additional, extraneous information about nodes and their relationships in order to rank nodes. Two solution concepts in particularCoalitional semivalues (Szczepański et al., 2014) and configuration semivalues (Tarkowski et al., 2016) - have already been used as game-theoretic centrality measures that incorporate an extraneously defined community structure in the ranking. Nodes benefit from belonging to important communities, or to a large number of communities. Other interesting directions for future research include whether the game-theoretic approach can also be used to analyse multi-layer networks (where multiple types of relationships may exist between nodes), hypergraphs (where relationships can exist between more than two nodes), or other types of networks.

Third, the axiomatic study of game-theoretic network centrality measures is still in its infancy. Such an analysis is very important in order to characterise and study the properties of such measures. Van den Brink and Gilles (2000) axiomatised their degree-based centrality measure andinterestingly - developed a closed-form formula for it that easily translates to a polynomial algorithm. Skibski, Michalak, and Rahwan (2018), Skibski, Rahwan, Michalak, and Yokoo (2016), and Skibski, and Sosnowska (2017) have also studied axiomatisation of game-theoretic network centrality, but did not analyse computational properties. It would be interesting to investigate whether there is any relation between the axioms and the computational properties of a given measure (e.g., a given axiom might be very desirable but too computationally complex to implement).

Finally, the study of the applications of game-theoretic network centrality and the evaluation of the quality and comparative studies of network centrality measures in general require more attention.

\section{Acknowledgements}

Paul Harrenstein, Tomasz Michalak and Michael Wooldridge were supported by the European Research Council under Advanced Grant 291528 ("RACE"). This work was also supported by the Polish National Science Centre grant DEC-2013/09/D/ST6/03920.

\section{Appendix A. Summary of Main Notation}

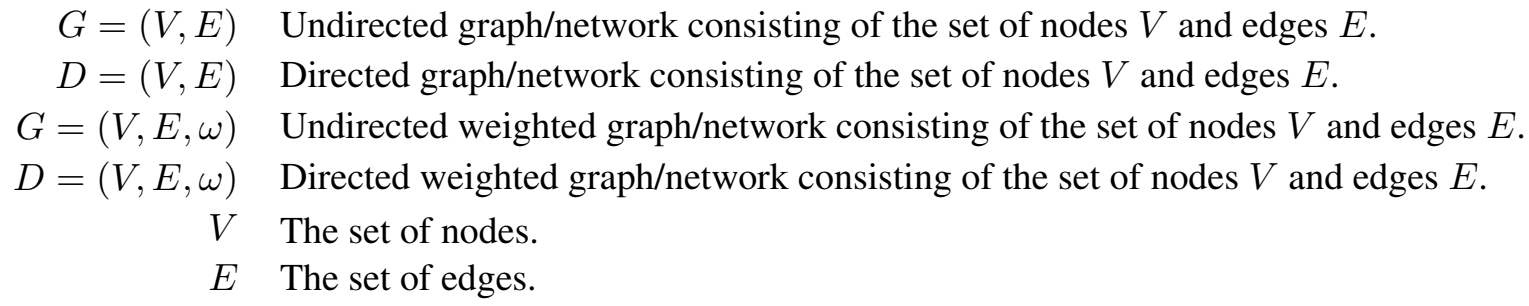




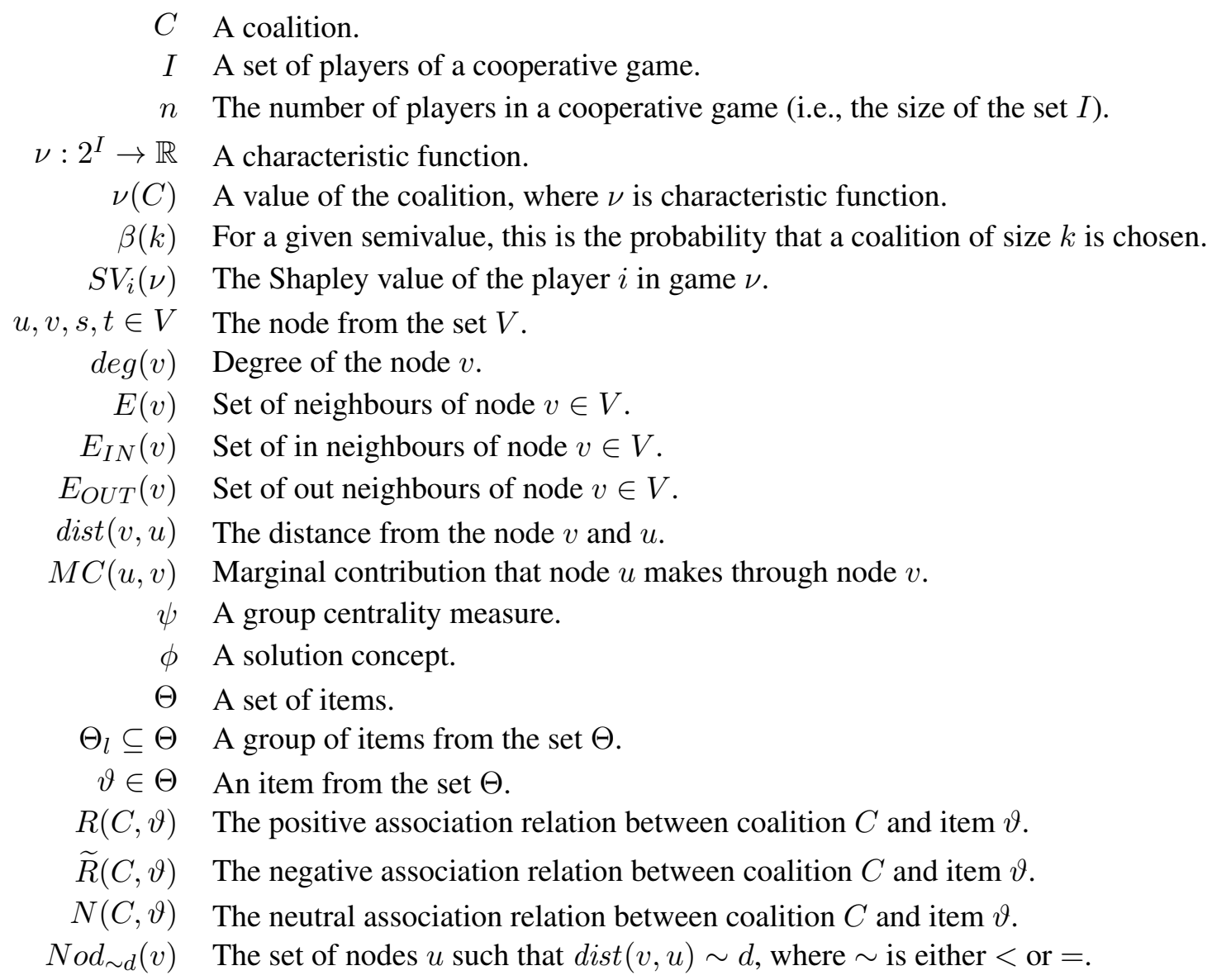

\section{Appendix B. Algorithms for Section 6}

In this appendix, we present the algorithms from Section 6. Each of the algorithms makes use of Algorithm 1 and passes to it the required arguments in order to compute the respective centrality measure.

\section{B.1 Semivalue Degree Algorithm}

Algorithm 3 computes the semivalue degree centrality. 


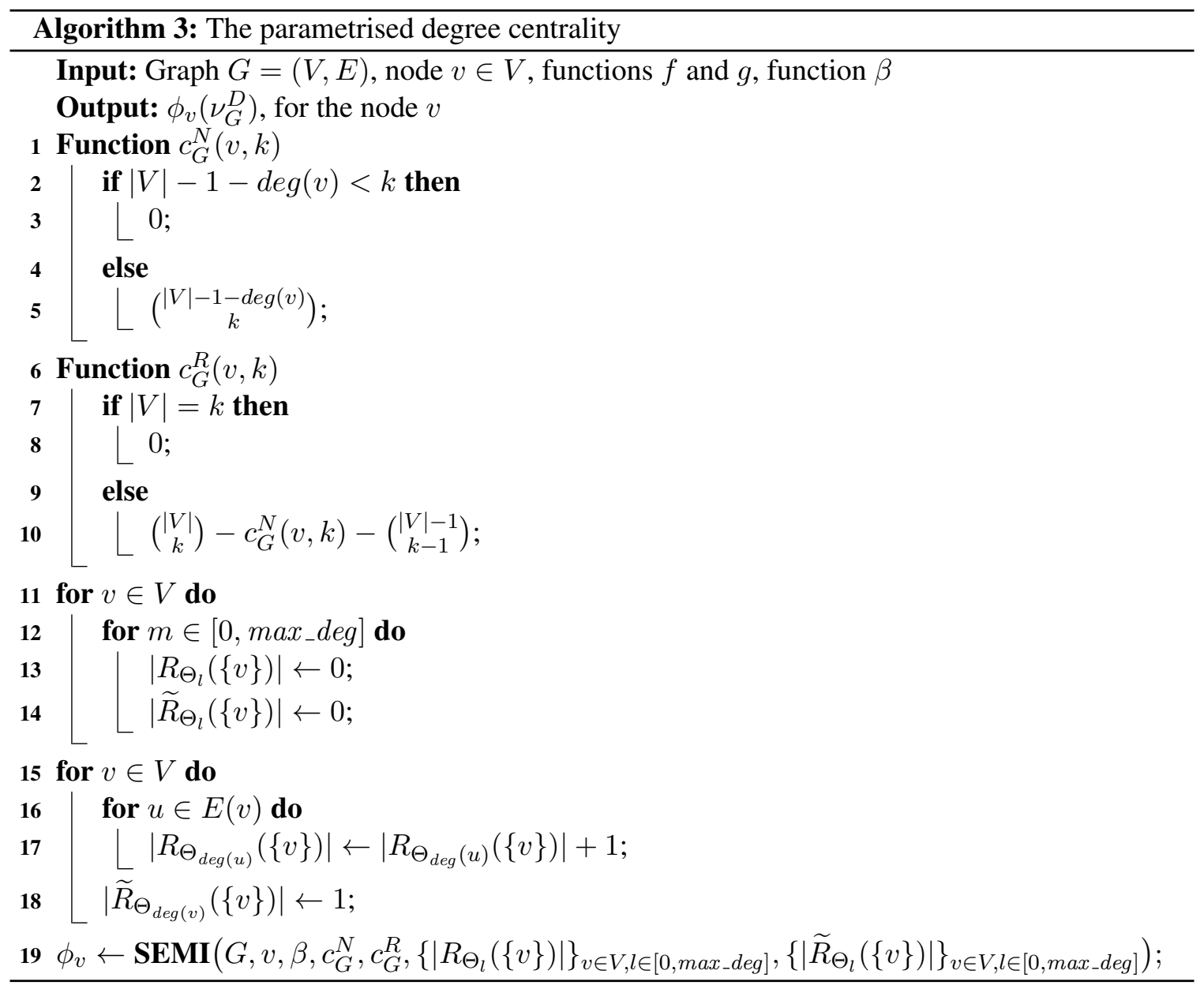

\section{B.2 Semivalue Betweenness Algorithm}

Let us discuss how to compute the semivalue betweenness centrality. First, we show in Algorithm 4 how to count the number of shortest paths between each pair of nodes and the number of paths that pass through a given node. Next, in Algorithm 5 we present the main computations. 


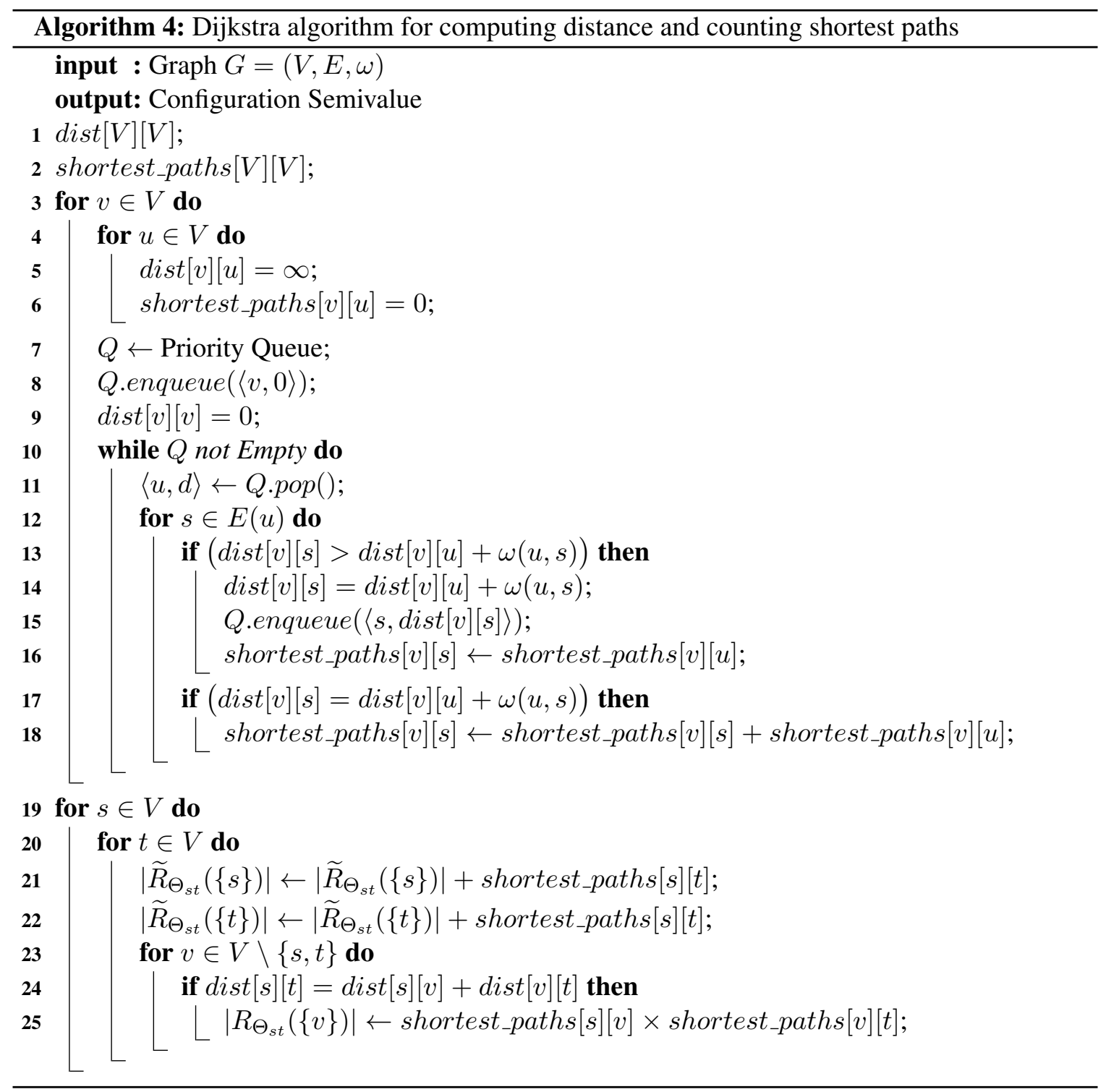




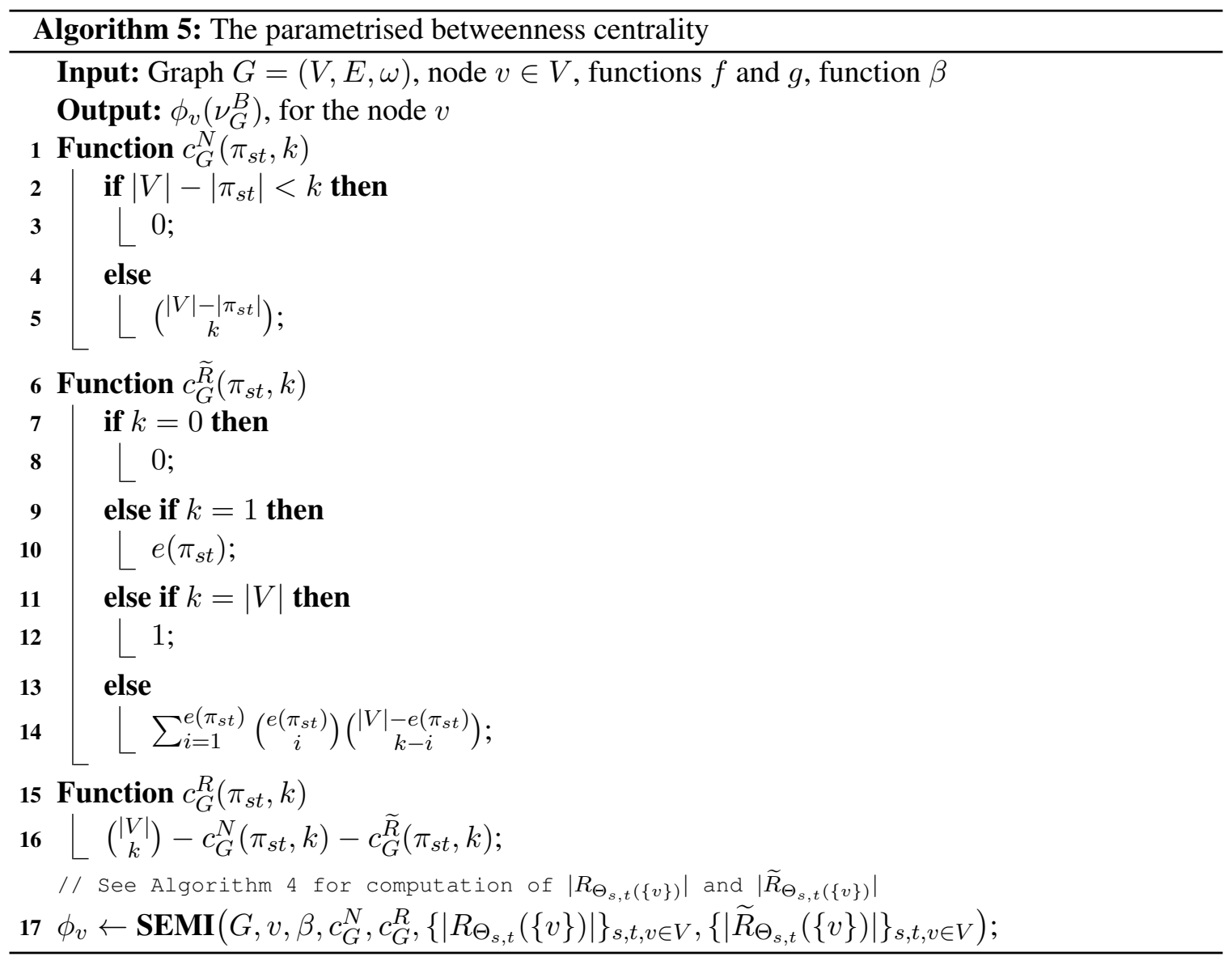

\section{B.3 Semivalue Closeness Algorithm}

Let us present Algorithm 6 for computing the semivalue closeness centrality. First, however, we will improve computation of $\left|R_{\# k}^{-1}(\langle u, d\rangle)\right|$ and $\left|N_{\# k}^{-1}(\langle u, d\rangle)\right|$ from $O(|V|)$ to $O(1)$. Assuming $\operatorname{Nod}_{=d}(u)>0$ (otherwise $\left|R_{\# k}^{-1}(\langle u, d\rangle)\right|$ is equal to 0 ), we can use the well-known combinatoric property, $\sum_{0 \leq i \leq m}\left(\begin{array}{c}m \\ i\end{array}\right)\left(\begin{array}{c}n-m \\ k-i\end{array}\right)=\left(\begin{array}{c}n \\ k\end{array}\right)$, to simplify:

$$
\left|R_{\# k}^{-1}(\langle u, d\rangle)\right|=\left(\begin{array}{c}
|V|-N_{o d}(u) \\
k
\end{array}\right)-\left(\begin{array}{c}
|V|-N o d_{=d}(u)-N_{o d}(u) \\
k
\end{array}\right)
$$

Next, using similar techniques we can simplify $\left|\widetilde{R}_{\# k}^{-1}(\langle u, d\rangle)\right|$ as follows:

$$
\left|\widetilde{R}_{\# k}^{-1}(\langle u, d\rangle)\right|=\left(\begin{array}{c}
|V| \\
k
\end{array}\right)-\left(\begin{array}{c}
|V|-N_{o d}(u) \\
k
\end{array}\right)
$$




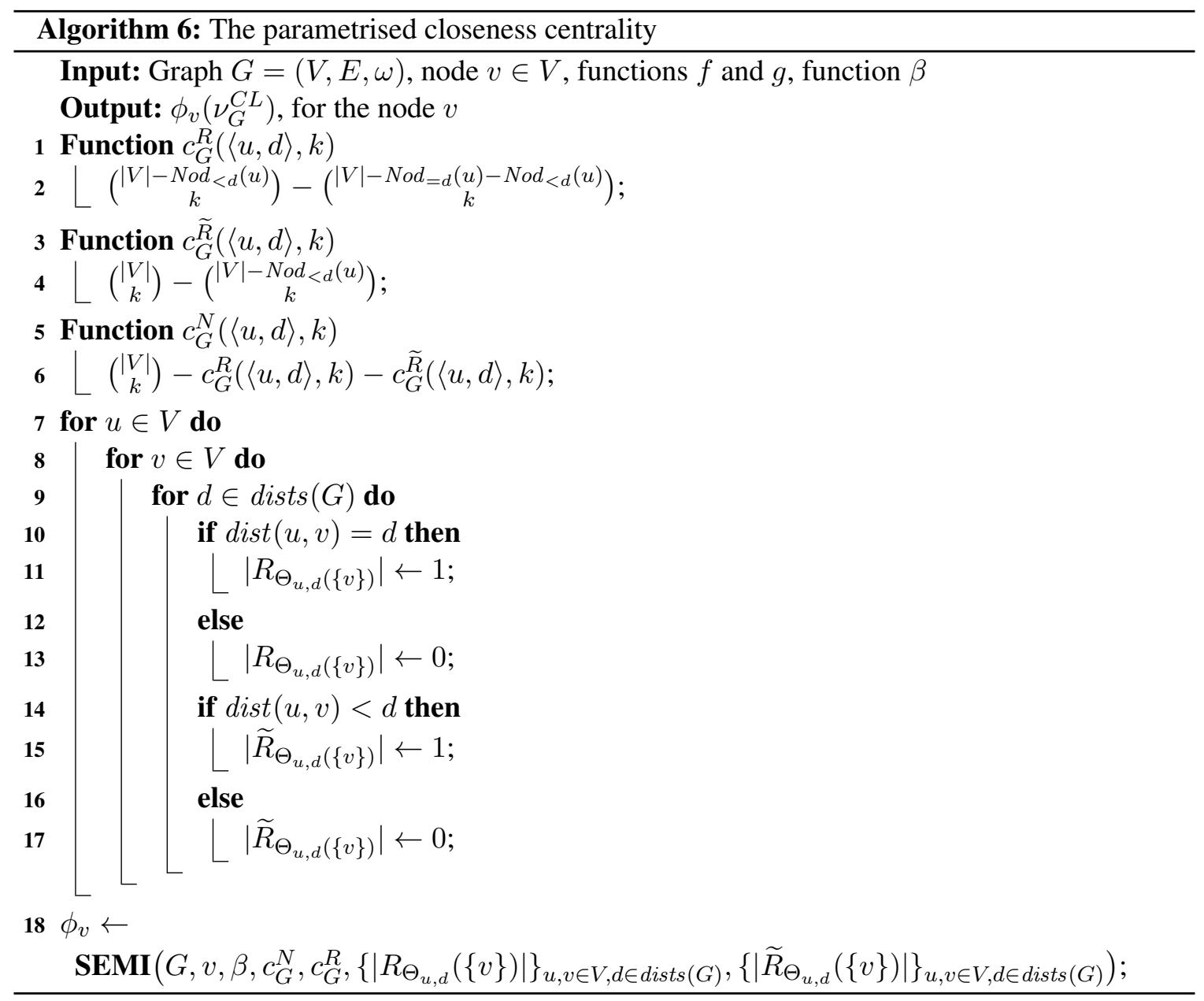

\section{Appendix C. Proofs for Section 7}

In this section, we present the proofs for the derivations of the Banzhaf index algorithms in Section 7.

\section{C.1 General Derivations for the Banzhaf Index}

In this section, we will reorder Equation 7 in order to group all terms with the variable $k$ in one spot. This will allow us to get rid of the variable altogether in the next sections, yielding faster computation. 
Proof: From Theorem 1 we have: ${ }^{10}$

$$
\begin{aligned}
& \phi_{v}(\nu) \\
= & \sum_{0 \leq k<|V|} \beta(k) \sum_{1 \leq l \leq h(G)} \frac{\mathrm{MC}\left(k, u, \Theta_{l}\right)}{\left(\begin{array}{c}
|V|-1 \\
k
\end{array}\right)} . \\
= & \sum_{0 \leq k<|V|} \beta(k) \sum_{1 \leq l \leq h(G)} \frac{\left|R_{\Theta_{l}}(\{u\})\right| \mathrm{MC}^{[1]}\left(k, u, \Theta_{l}\right)}{\left(\begin{array}{c}
|V|-1 \\
k
\end{array}\right)} \\
& -\sum_{0 \leq k<|V|} \beta(k) \sum_{1 \leq l \leq h(G)} \frac{\left|\widetilde{R}_{\Theta_{l}}(\{u\})\right| \mathrm{MC}^{[2]}\left(k, u, \Theta_{l}\right)}{\left(\begin{array}{c}
|V|-1 \\
k
\end{array}\right)} \\
= & \sum_{0 \leq k<|V|} \beta(k) \sum_{1 \leq l \leq h(G)} \frac{\left|R_{\Theta_{l}}(\{u\})\right| f\left(\Theta_{l}\right)\left|N_{\# k}^{-1}\left(\Theta_{l}\right)\right|}{\left(\begin{array}{l}
|V|-1 \\
k
\end{array}\right)} \\
& -\sum_{0 \leq k<|V|} \beta(k) \sum_{1 \leq l \leq h(G)} \frac{\left|\widetilde{R}_{\Theta_{l}}(\{u\})\right| f\left(\Theta_{l}\right)\left|R_{\# k}^{-1}\left(\Theta_{l}\right)\right|}{\left(\begin{array}{l}
|V|-1 \\
k
\end{array}\right)} \\
= & \sum_{1 \leq l \leq h(G)}\left|R_{\Theta_{l}}(\{u\})\right| f\left(\Theta_{l}\right) \sum_{0 \leq k<|V|} \beta(k) \frac{\left|N_{\# k}^{-1}\left(\Theta_{l}\right)\right|}{\left(\begin{array}{c}
|V|-1 \\
k
\end{array}\right)} \\
& -\sum_{1 \leq l \leq h(G)}\left|\widetilde{R}_{\Theta_{l}}(\{u\})\right| f\left(\Theta_{l}\right) \sum_{0 \leq k<|V|} \beta(k) \frac{\left|R_{\# k}^{-1}\left(\Theta_{l}\right)\right|}{\left(\begin{array}{l}
|V|-1 \\
k
\end{array}\right)} \\
= & \sum_{1 \leq l \leq h(G)}\left|R_{\Theta_{l}}(\{u\})\right| f\left(\Theta_{l}\right) \mathcal{N}^{l}-\sum_{1 \leq l \leq h(G)} \frac{\left|\widetilde{R}_{\Theta_{l}}(\{u\})\right| f\left(\Theta_{l}\right) \mathcal{R}^{l}}{\sum_{l}(G)}
\end{aligned}
$$

\section{C.2 Derivations for Banzhaf Degree Centrality}

In this section, we prove Proposition 4.

Proof: Recall that:

$$
\left|N_{\# k}^{-1}(v)\right|= \begin{cases}0 & \text { if }|V|-1-\operatorname{deg}(v)<k \\
\left(\begin{array}{c}
|V|-1-\operatorname{deg}(v) \\
k
\end{array}\right) & \text { otherwise. }\end{cases}
$$

and

$$
\left|R_{\# k}^{-1}(v)\right|= \begin{cases}0 & \text { if }|V|=k \\
\left(\begin{array}{c}
|V| \\
k
\end{array}\right)-\left|N_{\# k}^{-1}(v)\right|-\left(\begin{array}{c}
|V|-1 \\
k-1
\end{array}\right) & \text { otherwise. }\end{cases}
$$

10. Note that $\operatorname{MC}^{[3]}\left(k, u, \Theta_{l}\right)=0$ since $\Delta^{k}=g(k+1)-g(k)=0$ and it is therefore omitted in the below formula 
Using the property $\sum_{0 \leq k \leq n}\left(\begin{array}{l}n \\ k\end{array}\right)=2^{n}$ we have:

$$
\begin{aligned}
\mathcal{N}^{l} & =\sum_{0 \leq k<|V|} \beta(k) \frac{\left|N_{\# k}^{-1}\left(\Theta_{l}\right)\right|}{\left(\begin{array}{c}
|V|-1 \\
k
\end{array}\right)} \\
\mathcal{N}^{\operatorname{deg}(v)} & =\frac{1}{2^{|V|-1}} \sum_{0 \leq k<|V|}\left(\begin{array}{c}
|V|-1-\operatorname{deg}(v) \\
k
\end{array}\right)=\frac{2^{|V|-1-\operatorname{deg}(v)}}{2^{|V|-1}}=\frac{1}{2^{\operatorname{deg}(v)}},
\end{aligned}
$$

and

$$
\begin{aligned}
\mathcal{R}^{l} & =\sum_{0 \leq k<|V|} \beta(k) \frac{\left|R_{\# k}^{-1}\left(\Theta_{l}\right)\right|}{\left(\begin{array}{c}
|V|-1 \\
k
\end{array}\right)} \\
\mathcal{R}^{\operatorname{deg}(v)} & =\frac{1}{2^{|V|-1}} \sum_{0 \leq k<|V|}\left(\begin{array}{c}
|V|-1 \\
k
\end{array}\right)-\left(\begin{array}{c}
|V|-1-\operatorname{deg}(v) \\
k
\end{array}\right)=1-\frac{1}{2^{\operatorname{deg}(v)}}
\end{aligned}
$$

Since $h(G)=|V|, \mathcal{N}^{l}$ and $\mathcal{R}^{l}$ can be precomputed in constant time, and $R_{\Theta_{l}}(\{v\})$ and $\widetilde{R}_{\Theta_{l}}(\{v\})$ can be computed in $O(|V|+|E|)$ time, then the Banzhaf generalised degree centrality (with $g(|C|)=$ 1) can be computed by Algorithm 2 in $O\left(|V|^{1+m_{1}}+|E|\right)$ time.

\section{C.3 Derivations for Banzhaf Betweenness Centrality}

In this section, we prove Proposition 5.

Proof: Recall that:

$$
\left|N_{\# k}^{-1}\left(\pi_{s t}\right)\right|= \begin{cases}0 & \text { if }|V|-\left|\pi_{s t}\right|<k \\
\left(\begin{array}{c}
|V|-\left|\pi_{s t}\right| \\
k
\end{array}\right) & \text { otherwise. }\end{cases}
$$

and

$$
\left|\widetilde{R}_{\# k}^{-1}\left(\pi_{s t}\right)\right|= \begin{cases}0 & \text { if } k=0 \\
e\left(\pi_{s t}\right) & \text { if } k=1 \\
1 & \text { if } k=|V| \\
\sum_{i=1}^{e\left(\pi_{s t}\right)}\left(\begin{array}{c}
e\left(\pi_{s t}\right) \\
i
\end{array}\right)\left(\begin{array}{c}
|V|-e\left(\pi_{s t}\right) \\
k-i
\end{array}\right) & \text { otherwise, }\end{cases}
$$

where $e\left(\pi_{s t}\right)=\min \left(2,\left|\pi_{s t}\right|\right)$, and

$$
\left|R_{\# k}^{-1}\left(\pi_{s t}\right)\right|=\left(\begin{array}{c}
|V| \\
k
\end{array}\right)-\left|\widetilde{R}_{\# k}^{-1}\left(\pi_{s t}\right)\right|-\left|N_{\# k}^{-1}\left(\pi_{s t}\right)\right| .
$$

We can now calculate values:

$$
\begin{aligned}
\mathcal{N}^{l} & =\sum_{0 \leq k<|V|} \beta(k) \frac{\left|N_{\# k}^{-1}\left(\Theta_{l}\right)\right|}{\left(\begin{array}{c}
|V|-1 \\
k
\end{array}\right)} \\
\mathcal{N}^{s, t} & =\frac{1}{2^{|V|-1}} \sum_{0 \leq k<|V|}\left(\begin{array}{c}
|V|-\left|\pi_{s t}\right| \\
k
\end{array}\right) \\
& =\frac{2^{|V|-\left|\pi_{s t}\right|}}{2^{|V|-1}}
\end{aligned}
$$


and define

$$
\begin{aligned}
\widetilde{\mathcal{R}}^{l} & =\sum_{0 \leq k<|V|} \beta(k) \frac{\left|R_{\# k}^{-1}\left(\Theta_{l}\right)\right|}{\left(\begin{array}{c}
|V|-1 \\
k
\end{array}\right)} \\
\widetilde{\mathcal{R}}^{s, t} & =\frac{1}{2^{|V|-1}}\left(e\left(\pi_{s t}\right)+1+\sum_{2 \leq k<|V|-1} \sum_{i=1}^{e\left(\pi_{s t}\right)}\left(\begin{array}{c}
e\left(\pi_{s t}\right) \\
i
\end{array}\right)\left(\begin{array}{c}
|V|-e\left(\pi_{s t}\right) \\
k-i
\end{array}\right)\right)
\end{aligned}
$$

Assuming $e\left(\pi_{s t}\right)=2$ we have:

$$
\begin{aligned}
& =\frac{1}{2^{|V|-1}}\left(3+2 \sum_{2 \leq k<|V|-1}\left(\begin{array}{c}
|V|-2 \\
k-1
\end{array}\right)+\sum_{2 \leq k<|V|-1}\left(\begin{array}{c}
|V|-2 \\
k-2
\end{array}\right)\right) \\
& =\frac{1}{2^{|V|-1}}\left(3\left(2^{|V|-2}\right)-|V|\right)
\end{aligned}
$$

Assuming $e\left(\pi_{s t}\right)=1$, we get the following:

$$
\begin{aligned}
& =\frac{1}{2^{|V|-1}}\left(2+\sum_{2 \leq k<|V|-1}\left(\begin{array}{c}
|V|-2 \\
k-1
\end{array}\right)\right) \\
& =\frac{2^{|V|-2}}{2^{|V|-1}} \\
& =\frac{1}{2}
\end{aligned}
$$

Finally, we have

$$
\mathcal{R}^{s, t}=\frac{2^{|V|}-1}{2^{|V|-1}}-\widetilde{\mathcal{R}}^{s, t}-\mathcal{N}^{s, t}
$$

Since $h(G)=|V|^{2}, \mathcal{N}^{l}$ and $\mathcal{R}^{l}$ can be computed in constant time, and $R_{\Theta_{s, t}}(\{v\})$ and $\widetilde{R}_{\Theta_{s, t}}(\{v\})$ can be precomputed in $O\left(|V|^{2}+|V||E|\right)$ time for undirected graphs and $O\left(|V||E|+|V|^{2} \log (|V|)\right)$ time for directed graphs, then the Banzhaf generalised betweenness centrality (with $g(|C|)=$ 1) can be computed by Algorithm 2 in $O\left(|V|^{2+m_{1}}+|V||E|\right)$ time for undirected graphs, and $O\left(|V|^{2+m_{1}}+|V||E|+|V|^{2} \log (|V|)\right)$ time for directed graphs.

\section{C.4 Derivations for Banzhaf Closeness Centrality}

In this section, we prove Proposition 6.

Proof: Recall that:

$$
\begin{aligned}
& \left|R_{\# k}^{-1}(\langle u, d\rangle)\right|=\sum_{i=1}^{N o d_{=d}(u)}\left(\begin{array}{c}
N o d_{=d}(u) \\
i
\end{array}\right)\left(\begin{array}{c}
|V|-N o d_{=d}(u)-N_{<d}(u) \\
k-i
\end{array}\right), \\
& \left|\widetilde{R}_{\# k}^{-1}(\langle u, d\rangle)\right|=\sum_{i=1}^{N o d_{<d}(u)}\left(\begin{array}{c}
N o d_{<d}(u) \\
i
\end{array}\right)\left(\begin{array}{c}
|V|-N_{<d}(u) \\
k-i
\end{array}\right)
\end{aligned}
$$


and

$$
\begin{gathered}
\left|N_{\# k}^{-1}(\langle u, d\rangle)\right|=\left(\begin{array}{c}
|V| \\
k
\end{array}\right)-\left|R_{\# k}^{-1}(\langle u, d\rangle)\right|-\left|\widetilde{R}_{\# k}^{-1}(\langle u, d\rangle)\right| . \\
\mathcal{R}^{l}=\sum_{0 \leq k<|V|} \beta(k) \frac{\left|R_{\# k}^{-1}\left(\Theta_{l}\right)\right|}{\left(\begin{array}{c}
|V|-1 \\
k
\end{array}\right)} \\
\mathcal{R}^{u, d}=\frac{1}{2^{|V|-1}} \sum_{0 \leq k<|V|} \sum_{i=1}^{N o d_{=d}(u)}\left(\begin{array}{c}
N o d_{=d}(u) \\
i
\end{array}\right)\left(\begin{array}{c}
|V|-N_{=d}(u)-\operatorname{Nod}_{<d}(u) \\
k-i
\end{array}\right)
\end{gathered}
$$

Assuming $\operatorname{Nod}_{=d}(u)>0$ (otherwise the value is equal to 0 ), we can use the well-known combinatoric property $\sum_{0 \leq i \leq m}\left(\begin{array}{c}m \\ i\end{array}\right)\left(\begin{array}{c}n-m \\ k-i\end{array}\right)=\left(\begin{array}{l}n \\ k\end{array}\right)$ to simplify:

$$
=\frac{1}{2^{|V|-1}}\left(\sum_{0 \leq k<|V|}\left(\begin{array}{c}
|V|-N_{<d}(u) \\
k
\end{array}\right)-\sum_{0 \leq k<|V|}\left(\begin{array}{c}
|V|-N_{<d}(u)-N_{k} d_{<d}(u) \\
k
\end{array}\right)\right)
$$

Assuming $\operatorname{Nod}_{<d}(u)>0$ we have:

$$
=\frac{1}{2^{|V|-1}}\left(2^{|V|-N_{o d}(u)}-2^{|V|-N o d_{=d}(u)-N_{o d}<d}(u)\right)
$$

and assuming $\operatorname{Nod}_{<d}(u)=0$ we have:

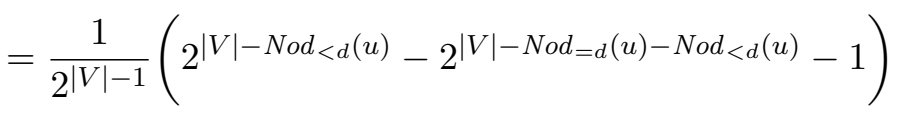

Next, let us define:

$$
\begin{aligned}
\widetilde{\mathcal{R}}^{u, d} & =\sum_{0 \leq k<|V|} \beta(k) \frac{\left|\widetilde{R}_{\# k}^{-1}\left(\Theta_{u, d}\right)\right|}{\left(\begin{array}{c}
|V|-1 \\
k
\end{array}\right)} \\
& =\frac{1}{2^{|V|-1}} \sum_{0 \leq k<|V|} \sum_{i=1}^{N o d_{<d}(u)}\left(\begin{array}{c}
N o d_{<d}(u) \\
i
\end{array}\right)\left(\begin{array}{c}
|V|-N^{N} d_{<d}(u) \\
k-i
\end{array}\right)
\end{aligned}
$$

We can simplify this value as above, which leads to the following result:

$$
\begin{aligned}
& =\frac{1}{2^{|V|-1}} \sum_{0 \leq k<|V|}\left(\begin{array}{c}
|V| \\
k
\end{array}\right)-\left(\begin{array}{c}
|V|-N_{0 d_{<d}(u)} \\
k
\end{array}\right) \\
& =\frac{1}{2^{|V|-1}}\left(2^{|V|}-1-\sum_{0 \leq k<|V|}\left(\begin{array}{c}
|V|-N_{<d}(u) \\
k
\end{array}\right)\right) .
\end{aligned}
$$


Again, assuming $\operatorname{Nod}_{<d}(u)=0$ we have:

$$
=\frac{1}{2^{|V|-1}}\left(2^{|V|}-1-2^{|V|-N_{<d}(u)}\right) .
$$

and assuming $\operatorname{Nod}_{<d}(u)>0$ we have:

$$
=\frac{1}{2^{|V|-1}}\left(2^{|V|}-2^{|V|-N_{o d}(u)}\right) .
$$

With this, we have all the tools to complete our proof:

$$
\begin{aligned}
\mathcal{N}^{l} & =\sum_{0 \leq k<|V|} \beta(k) \frac{\left|N_{\# k}^{-1}\left(\Theta_{l}\right)\right|}{\left(\begin{array}{c}
|V|-1 \\
k
\end{array}\right)} \\
\mathcal{N}^{u, d} & =\frac{2^{|V|}-1}{2^{|V|-1}}-\mathcal{R}^{u, d}-\widetilde{\mathcal{R}}^{u, d}
\end{aligned}
$$

Since $h(G) \cong O\left(|V|^{2}\right), \mathcal{N}^{l}$ and $\mathcal{R}^{l}$ can be computed in constant time, and $R_{\Theta_{u, d}}(\{v\})$ and $\widetilde{R}_{\Theta_{u, d}}(\{v\})$ can be precomputed in $O\left(|V|^{2}+|V||E|\right)$ time for undirected graphs and $O(|V||E|+$ $\left.|V|^{2} \log (|V|)\right)$ time for directed graphs, then the Banzhaf generalised closeness centrality (with $g(|C|)=1)$ can be computed by Algorithm 2 in $O\left(|V|^{2+m_{1}}+|V||E|\right)$ time for undirected graphs, and $O\left(|V|^{2+m_{1}}+|V||E|+|V|^{2} \log (|V|)\right)$ time for directed graphs.

\section{References}

Amer, R., \& Giménez, J. M. (2004). A connectivity game for graphs. Mathematical Methods of Operations Research, 60(3), 453-470.

Amer, R., Giménez, J., \& Magaña, A. (2012). Accessibility measures to nodes of directed graphs using solutions for generalized cooperative games. Mathematical Methods of Operations Research, 75(1), 105-134.

Aziz, H., \& de Keijzer, B. (2014). Shapley meets Shapley. In Mayr, E. W., \& Portier, N. (Eds.), 31 st International Symposium on Theoretical Aspects of Computer Science (STACS 2014), Vol. 25, pp. 99-111.

Bachrach, Y., \& Rosenschein, J. S. (2009). Power in threshold network flow games. Autonomous Agents and Multi-Agent Systems, 18(1), 106-132.

Banzhaf, J. F. (1965). Weighted Voting Doesn't Work: A Mathematical Analysis. Rutgers Law Review, 19, 317-343.

Boldi, P., \& Vigna, S. (2014). Axioms for centrality. Internet Mathematics, 10(3-4).

Bollen, J., Sompel, H. V., Smith, J. A., \& Luce, R. (2005). Toward alternative metrics of journal impact: A comparison of download and citation data. Information Processing and Management, 41(6), 1419-1440.

Bonacich, P. (1972). Factoring and weighting approaches to status scores and clique identification. Journal of Mathematical Sociology, 2(1), 113-120. 
Brandes, U. (2008). On variants of shortest-path betweenness centrality and their generic computation. Social Networks, 30(2), 136-145.

van den Brink, R., \& Borm, P. (2002). Digraph competitions and cooperative games. Theory and Decision, 53(4), 327-342.

van den Brink, R., \& Gilles, R. P. (2000). Measuring domination in directed networks. Social Networks, 22(2), 141-157.

Chalkiadakis, G., Elkind, E., \& Wooldridge, M. (2012). Computational Aspects of Cooperative Game Theory. Synthesis Lectures on Artificial Intelligence and Machine Learning. Morgan \& Claypool.

Cormen, T. H., Stein, C., Rivest, R. L., \& Leiserson, C. E. (2001). Introduction to Algorithms (2nd edition). McGraw-Hill Higher Education.

Deng, X., \& Papadimitriou, C. (1994). On the complexity of cooperative solution concepts. Mathematics of Operations Research, 19(2), 257-266.

Dijkstra, E. W. (1959). A note on two problems in connexion with graphs. Numerische Mathematik, $1(1), 269-271$.

Dubey, P., Neyman, A., \& Weber, R. J. (1981). Value theory without efficiency. Mathematics of Operations Research, 6(1), 122-128.

Elkind, E., Goldberg, L. A., Goldberg, P. W., \& Wooldridge, M. (2009). A tractable and expressive class of marginal contribution nets and its applications. Mathematical Logic Quarterly, 55(4), 362-376.

Everett, M. G., \& Borgatti, S. P. (1999). The centrality of groups and classes. Journal of Mathematical Sociology, 23(3), 181-201.

Freeman, L. C. (2004). The Development of Social Network Analysis: A Study in the Sociology of Science. Empirical Press.

Freeman, L. (1979). Centrality in social networks: Conceptual clarification. Social Networks, 1(3), 215-239.

Gillies, D. B. (1959). Solutions to general non-zero-sum games. Contributions to the Theory of Games, 4(40), 47-85.

Gillies, D. B. (1953). Some theorems on n-person games. Ph.D. thesis, Princeton University.

Girvan, M., \& Newman, M. E. (2002). Community structure in social and biological networks. Proceedings of the national academy of sciences, 99(12), 7821-7826.

Gómez, D., González-Arangüena, E., Manuel, C., Owen, G., Del Pozo, M., \& Tejada, J. (2003). Centrality and power in social networks: A game theoretic approach. Mathematical Social Sciences, 46(1), 27-54.

Greco, G., Malizia, E., Palopoli, L., \& Scarcello, F. (2009). On the complexity of compact coalitional games. In Boutilier, C. (Ed.), Proceedings of the Twenty First International Joint Conference on Artifical Intelligence (IJCAI '09), pp. 147-152.

Greco, G., Malizia, E., Palopoli, L., \& Scarcello, F. (2011). On the complexity of the core over coalition structures. In Walsh, T. (Ed.), Proceedings of the Twenty-Second International Joint Conference on Artificial Intelligence (IJCAI'11), pp. 216-221. Morgan Kaufmann. 
Grofman, B., \& Owen, G. (1982). A game-theoretic approach to measuring centrality in social networks. Social Networks, 4, 213-224.

Ieong, S., \& Shoham, Y. (2005). Marginal contribution nets: a compact representation scheme for coalitional games. In Proceedings of the 6th ACM conference on Electronic Commerce (ACM-EC'05), pp. 193-202. ACM.

Jeong, H., Mason, S. P., Barabasi, A. L., \& Oltvai, Z. N. (2001). Lethality and centrality in protein networks. Nature, 411(6833), 41-42.

Kempe, D., Kleinberg, J., \& Tardos, E. (2003). Maximizing the spread of influence through a social network. In Proceedings of the Ninth ACM SIGKDD International Conference on Knowledge Discovery and Data Mining (KDD'03), pp. 137-146, New York, NY, USA. ACM.

Kotter, R., Reid, A. T., Krumnack, A., Wanke, E., \& Sporns, O. (2007). Shapley ratings in brain networks.. Front Neuroinform, 1, 2.

Lindelauf, R., Hamers, H., \& Husslage, B. (2013). Cooperative game theoretic centrality analysis of terrorist networks: The cases of Jemaah Islamiyah and Al Qaeda. European Journal of Operational Research, 229(1), 230-238.

Maschler, M., Solan, E., \& Zamir, S. (2013). Game Theory. Cambridge University Press.

Michalak, T. P., Rahwan, T., Szczepański, P. L., Skibski, O., Narayanam, R., Wooldridge, M. J., \& Jennings, N. R. (2013a). Computational analysis of connectivity games with applications to the investigation of terrorist networks. In Rossi, F. (Ed.), Proceedings of the 23rd international joint conference on Artificial Intelligence (IJCAI'13), pp. 293-301. AAAI Press.

Michalak, T. P., Aadithya, K. V., Szczepanski, P. L., Ravindran, B., \& Jennings, N. R. (2013b). Efficient computation of the shapley value for game-theoretic network centrality. Journal of Artificial Intelligence Research (JAIR), 46, 607-650.

Monderer, D., \& Samet, D. (2002). Variations on the shapley value. In Aumann, R., \& Hart, S. (Eds.), Handbook of Game Theory with Economic Applications (1st edition)., Vol. 3, chap. 54, pp. 2055-2076. Elsevier.

Moreno, J. L. (1932). Application of the group method to classification. National Committee on Prisons and Prison Labor.

Moreno, J. L. (1934). Who shall survive? Nervous and Mental Disease Publishing Company.

Myerson, R. (1977). Graphs and cooperation in games. Mathematics of Operations Research, 2(3), 225-229.

Nebel, F. (2011). Graph-based coalitional games: An analysis via characteristics. In Proceedings of the 5th International ICST Conference on Performance Evaluation Methodologies and Tools (VALUETOOLS '11), pp. 476-485. ICST (Institute for Computer Sciences, SocialInformatics and Telecommunications Engineering), Brussels, Belgium.

Newman, M. E. J. (2004). Analysis of weighted networks. Physical Review E, 70(5), 056131.

Owen, G. (1977). Values of games with a priori unions. In Mathematical economics and game theory: Essays in honor of Oskar Morgenstern, Vol. 141 of Lecture Notes in Economics and Mathematical Systems, pp. 76-88. Springer. 
Page, L., Brin, S., Motwani, R., \& Winograd, T. (1999). The PageRank citation ranking: Bringing order to the web.. Technical report 1999-66, Stanford InfoLab.

Papadimitriou, C. H. (2003). Computational complexity. John Wiley and Sons Ltd.

Ramasuri, N., \& Narahari, Y. (2008). Determining the top- $k$ nodes in social networks using the Shapley value. In Padgham, L., Parkes, D., Müller, J. P., \& Parsons, S. (Eds.), Proceedings of the 7th International Conference on Autonomous Agents and Multi-Agent Systems (AA$\left.M A S^{\prime} 08\right)$, pp. 1509-1512. IFAAMAS.

Schmeidler, D. (1969). The nucleolus of a characteristic function game. SIAM Journal on applied mathematics, 17(6), 1163-1170.

Shapley, L. S. (1953). A value for $n$-person games. In Kuhn, H., \& Tucker, A. (Eds.), Contributions to the Theory of Games II, Vol. 28 of Annals of Mathematical Studies, pp. 307-317. Princeton University Press.

Skibski, O., Michalak, T. P., Rahwan, T., \& Wooldridge, M. (2014). Algorithms for the Shapley and Myerson values in graph-restricted games. In Lomuscio, A., Scerri, P., Bazzan, A., \& Huhns, M. (Eds.), Proceedings of the 13th International Conference on Autonomous Agents and Multiagent Systems (AAMAS'14), pp. 197-204. IFAAMAS.

Skibski, O., Michalak, T. P., \& Rahwan, T. (2018). Axiomatic characterization of game-theoretic network centralities. Journal of Artificial Intelligence Research, (62), 33-68.

Skibski, O., Rahwan, T., Michalak, T. \& Yokoo, M. (2016). Attachment centrality: An axiomatic approach to connectivity in networks. Proceedings of the 2016 International Conference on Autonomous Agents \& Multiagent Systems (AAMAS'16), 168-176.

Skibski, O. \& Sosnowska, J. (2017). Attachment centrality for weighted graphs. Proceedings of the Twenty-Sixth International Joint Conference on Artificial Intelligence (IJCAI'17), 416-422.

Suri, N., \& Narahari, Y. (2010). A Shapley Value based approach to discover influential nodes in social networks. IEEE Transactions on Automation Science and Engineering, 99, 1-18.

Szczepański, P. L., Michalak, T. P., \& Rahwan, T. (2012). A new approach to betweenness centrality based on the Shapley value. In Conitzer, V., \& Winikoff, M. (Eds.), Proceedings of the 11th International Joint Conference on Autonomous Agents and Multi-Agent Systems (AAMAS'12), pp. 239-246. IFAAMAS.

Szczepański, P. L., Michalak, T. P., \& Rahwan, T. (2016). Efficient algorithms for game-theoretic betweenness centrality. Artificial Intelligence, 231, 39-63.

Szczepański, P. L., Michalak, T. P., \& Wooldridge, M. (2014). A centrality measure for networks with community structure based on a generalization of the Owen value. In Schaub, T., Friedrich, G., \& O'Sullivan, B. (Eds.), Proceedings of the 21st European Conference on Artificial Intelligence (ECAI'14), pp. 867-872.

Szczepański, P. L., Tarkowski, M. K., Michalak, T. P., Harrenstein, P., \& Wooldridge, M. (2015). Efficient computation of semivalues for game-theoretic network centrality. In Proceedings of the 29th AAAI Conference on Artificial Intelligence (AAAI'15), pp. 461-469.

Tarkowski, M. K., Szczepański, P. L., Rahwan, T., Michalak, T. P., \& Wooldridge, M. (2016). Closeness centrality for networks with overlapping community structure. In Proceedings of the 30th AAAI Conference on Artificial Intelligence (AAAI'16), pp. 622-629. 
Tijms, H. (2012). Understanding probability. Cambridge University Press.

Wooldridge, M., \& Dunne, P. (2006). On the computational complexity of coalitional resource games. Artificial Intelligence, 170(10), 835-871. 